ARTICLE

https://doi.org/10.1038/s41467-019-09197-8

\title{
A Hox-TALE regulatory circuit for neural crest patterning is conserved across vertebrates
}

Hugo J. Parker (1) 1, Bony De Kumar', Stephen A. Green², Karin D. Prummel ${ }^{3}$, Christopher Hess ${ }^{3}$, Charles K. Kaufman ${ }^{4}$, Christian Mosimann (10 ${ }^{3}$, Leanne M. Wiedemann (10) ${ }^{1,5}$, Marianne E. Bronner ${ }^{2}$ \& Robb Krumlauf ${ }^{1,6}$

In jawed vertebrates (gnathostomes), Hox genes play an important role in patterning head and jaw formation, but mechanisms coupling Hox genes to neural crest (NC) are unknown. Here we use cross-species regulatory comparisons between gnathostomes and lamprey, a jawless extant vertebrate, to investigate conserved ancestral mechanisms regulating Hox2 genes in NC. Gnathostome Hoxa2 and Hoxb2 NC enhancers mediate equivalent NC expression in lamprey and gnathostomes, revealing ancient conservation of Hox upstream regulatory components in NC. In characterizing a lamprey hox $\alpha 2 \mathrm{NC} /$ hindbrain enhancer, we identify essential Meis, Pbx, and Hox binding sites that are functionally conserved within Hoxa2/Hoxb2 NC enhancers. This suggests that the lamprey hox 2 enhancer retains ancestral activity and that Hoxa2/Hoxb2 NC enhancers are ancient paralogues, which diverged in hindbrain and NC activities. This identifies an ancestral mechanism for Hox2 NC regulation involving a Hox-TALE regulatory circuit, potentiated by inputs from Meis and $\mathrm{Pbx}$ proteins and Hox auto-/cross-regulatory interactions.

\footnotetext{
${ }^{1}$ Stowers Institute for Medical Research, Kansas City, MO 64110, USA. ${ }^{2}$ Division of Biology and Biological Engineering, California Institute of Technology, Pasadena, CA 91125, USA. ${ }^{3}$ Institute of Molecular Life Sciences, University of Zurich, 8057 Zürich, Switzerland. ${ }^{4}$ Division of Medical Oncology, Washington University in Saint Louis, St. Louis, MO 63110, USA. ${ }^{5}$ Department of Pathology and Laboratory Medicine, Kansas University Medical Center, Kansas City, KS 66160, USA. ${ }^{6}$ Department of Anatomy and Cell Biology, Kansas University Medical Center, Kansas City, KS 66160, USA. Correspondence and requests for materials should be addressed to M.E.B. (email: mbronner@caltech.edu) or to R.K. (email: rek@stowers.org)
} 
$\mathrm{N}$ eural crest $(\mathrm{NC})$ cells are a migratory and multipotent cell type, representing a defining characteristic of vertebrates $^{1-3}$. The cranial NC emerges from the mid/hindbrain region and contributes to cartilage and bone of the pharynx. Gnathostomes (jawed vertebrates) exhibit nested Hox gene expression domains (Hox codes) along the anteroposterior (AP) extent of both the neural tube and adjacent NC. However, little is known regarding shared versus independent regulation of Hox expression in these two tissues. In $\mathrm{NC}$ of the pharyngeal arches (PAs), the NC Hox code confers positional identity to each arch ${ }^{4}$. There is also evidence for an earlier role of Hox genes in regional generation of $\mathrm{NC}$ from the hindbrain ${ }^{4,5}$. Emergence of $\mathrm{NC}$ and its underlying Hox code played an important role in craniofacial evolution, giving rise to unique structures of the head and neck, including the jaws and hyoid apparatus used in feeding and respiration. Genome analyses together with gene expression and functional studies have described a common framework for a gene regulatory network (GRN) that drives NC induction, specification, and migration across vertebrates, and some regulatory components are present in non-vertebrate chordates ${ }^{1,6,7}$. However, Hox genes have not yet been integrated within the current formulation of the NC GRN. This is in part because the mechanisms regulating Hox expression in $\mathrm{NC}$ are relatively unclear compared to the current knowledge of Hox regulation in hindbrain segmentation ${ }^{4}$. Hence, despite the established functional roles of Hox genes in cranial $\mathrm{NC}^{4,8}$, whether the Hox code is coupled to this conserved NC GRN and if so how, is unknown. It is unclear whether Hox networks that control axial patterning and generation of $\mathrm{NC}$ are integrated within or are working in parallel, independently from the NC GRN.

Hox2 genes are critical components of the cranial NC Hox code and provide an important avenue for addressing this question. Experimental alterations in expression of gnathostome Hox2 paralogues (Hoxa2 and Hoxb2) lead to homeotic transformations. In mice, ectopic Hoxa2 suppresses jaw formation', while Hoxa2 mutants exhibit mirror image jaw duplication, indicating a role as a selector gene in NC AP identity ${ }^{4,8}$. Regulatory analyses in mouse, chick, and zebrafish have identified evolutionarily conserved enhancers in the Hoxa2 and the Hoxb2 genes that mediate their expression in $\mathrm{NC}$ and hindbrain segments (rhombomeres (r)) (Fig. 1a, b) ${ }^{4,10-13}$. Analyses of mouse Hoxa2 and Hoxb2 NC enhancers provide conflicting mechanisms for NC expression of Hox genes. A single $5^{\prime}$-flanking enhancer drives Hoxb2 in $\mathrm{r} 4$ and its $\mathrm{NC}^{11}$, supporting a model for shared regulation in these tissues (Fig. 1b). In contrast, independent exonic/intronic and $5^{\prime}$-flanking enhancers regulate Hoxa2 expression in $\mathrm{r}^{12}$ versus $\mathrm{r} 4$-derived $\mathrm{NC}^{14}$ (PA2), suggesting the evolution of distinct regulatory mechanisms between the hindbrain and NC (Fig. 1a). Characterisation of cis-elements required for the activity of these enhancers has provided some insight into their underlying regulatory mechanisms (Fig. 1a, b). Both the Hoxb2 r4/NC and the Hoxa2 exonic/intronic r4 enhancers depend upon the combined inputs from Meis and Pbx-Hox binding sites for their activities ${ }^{11,15-17}$. However, the inputs into the independent Hoxa2 $\mathrm{NC}$ enhancer are largely unknown, except for a binding site for transcription factor AP2- $\alpha$ (Tfap2 $\alpha$ ) in the mouse enhancer that is not conserved across gnathostome species ${ }^{10,12}$. Taken together, these analyses reveal differences between Hoxa2 and Hoxb2 in the enhancers and regulatory mechanisms underlying their expression in $\mathrm{r} 4$ and NC. Hence, in mouse, each gene supports a different model with respect to shared versus independent regulation in the hindbrain and NC. Given that Hoxa2 and Hoxb2 are paralogous genes, this raises two questions: what is the evolutionary relationship between their NC enhancers and which of these mechanisms is ancestral?
The sea lamprey offers an opportunity to address conserved ancestral mechanisms of Hox $2 \mathrm{NC}$ regulation in vertebrates. The extant jawless vertebrates (cyclostomes), lamprey and hagfish, represent a sister group to gnathostomes, so comparisons between these lineages can provide insights into the evolution of gene regulatory programs in vertebrates ${ }^{18-20}$. Such comparisons can reveal aspects of vertebrate biology that were present in the common ancestor of extant vertebrates and which have been conserved in each lineage. These studies can also identify features that differ between each lineage, which could represent divergence from the ancestral vertebrate state in either/both lineages. While comparisons between cyclostomes and gnathostomes may reveal ancestral features of vertebrate hox 2 regulation in the NC, little is known about the enhancers and regulatory factors underlying Hox NC expression in cyclostomes. Here, we employ cross-species regulatory comparisons between lamprey and gnathostomes to isolate and characterize NC enhancers and investigate ancestral regulation of Hox2 in vertebrates.

\section{Results}

Expression of hox 2 genes in lamprey cranial NC. In two species of lamprey, sea lamprey (Petromyzon marinus) and Arctic lamprey (Lethenteron camtschaticum), only two Hox2 paralogues (hox $\alpha 2$ and hox $\delta 2$ ) have been identified, but their orthology to gnathostome Hoxa2/Hoxb2 remains unresolved (Fig. 2a) ${ }^{21-23}$. The sea lamprey has six Hox clusters, compared to the four clusters inferred in ancestral gnathostomes ${ }^{22}$. A recent reconstruction based on comparisons of gene order at the chromosomal level between vertebrate species supports a model in which the ancestor of cyclostomes and gnathostomes also had four Hox clusters $^{22}$, suggesting that the two additional Hox clusters in lamprey arose from duplication event/s in the lamprey/cyclostome lineage. To investigate the duplication history of lamprey Hox clusters, previous work employed a chromosome-wide analysis of genomic synteny (duplicate gene retention) between lamprey Hox-bearing chromosomes ${ }^{22}$. These pairwise comparisons indicated that the chromosomes bearing the hox $\alpha$ and hox $\delta$ clusters display a significantly closer relationship to each other than to any other Hox-bearing chromosomes. Similarly, the chromosomes carrying the hox $\beta$ and hoxe clusters also show a significant enrichment for shared paralogues with each other. This suggests that these pairs of chromosomes derive from duplication event/s that occurred more recently than the duplication events that gave rise to the other Hox-bearing chromosomes ${ }^{22}$. Phylogenetic analyses reveal hox $\beta$ and hoxe paralogues consistently clustering in protein trees ${ }^{21,22}$, while hox $\alpha$ and hox $\delta$ paralogues do not show any clear patterns of clustering. This may be due to the limitations of phylogenetic analyses in resolving the relative timing of ancient duplication events ${ }^{22}$. Thus, the evidence from synteny analysis leads us to infer that hox $\alpha 2$ and hox $\delta 2$ genes are paralogues that arose from duplication in the lamprey/cyclostome lineage.

In exploring hox gene NC regulation in sea lamprey embryos, our time-course analysis of hox 1-3 expression revealed nested domains in the pharynx from stage (st) 23, reminiscent of those in Arctic lamprey ${ }^{24,25}$ and in gnathostomes ${ }^{4,8}$ (Fig. 2b-e). hox 2 is expressed in the NC posterior to PA1 and in the hindbrain posterior to $\mathrm{r} 1$, similar to gnathostome Hoxa2. In contrast, hox $\delta 2$ is expressed in $\mathrm{r} 3 / \mathrm{r} 5$, notochord, and in posterior pharyngeal endoderm and mesenchyme, but not at high levels in NC (Fig. 2b, c). If hox $\alpha / \delta$ clusters arose by duplication in the lamprey/cyclostome lineage, as supported by synteny analysis, this suggests that hox 22 expression diverged after this duplication. Given the similarity in $\mathrm{NC}$ expression of hox 2 to gnathostome Hoxa2, we focused our comparative regulatory analysis on mechanisms mediating lamprey hox 22 expression in NC. 
a

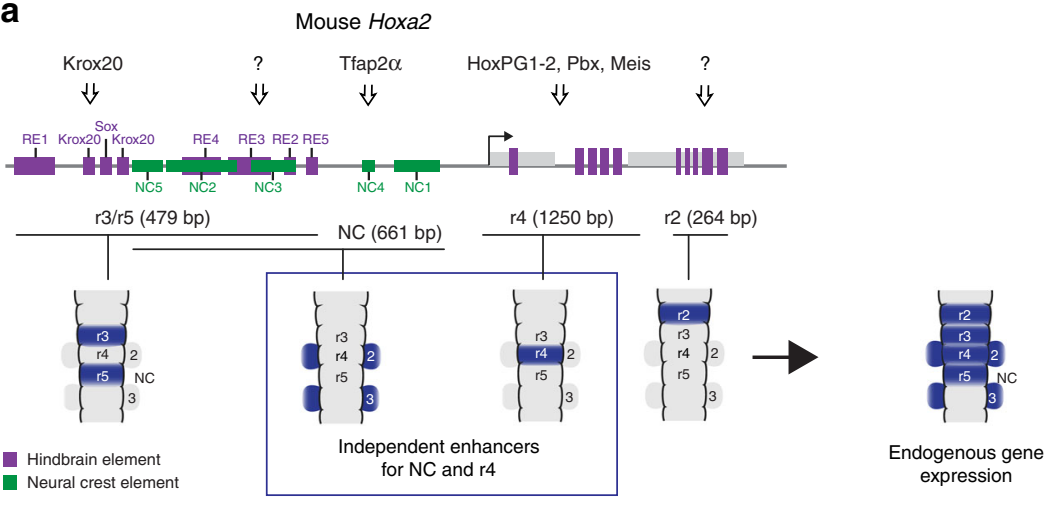

b

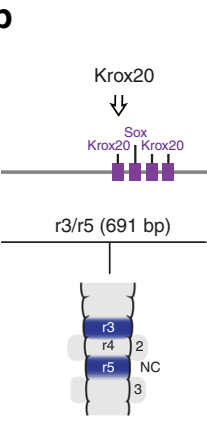

Mouse Hoxb2

Hoxb1, Pbx, Meis

V

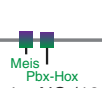

r4 + NC (181 bp)
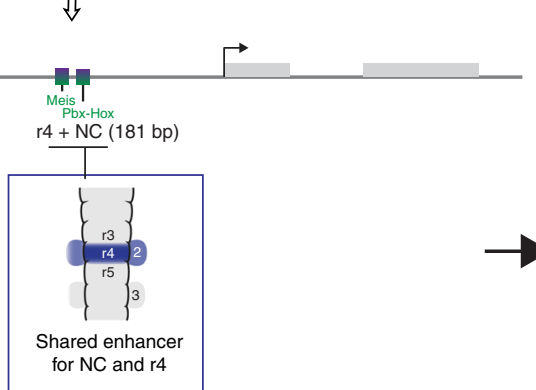

(anprey hoxis

c

Lamprey hox $\alpha 2$
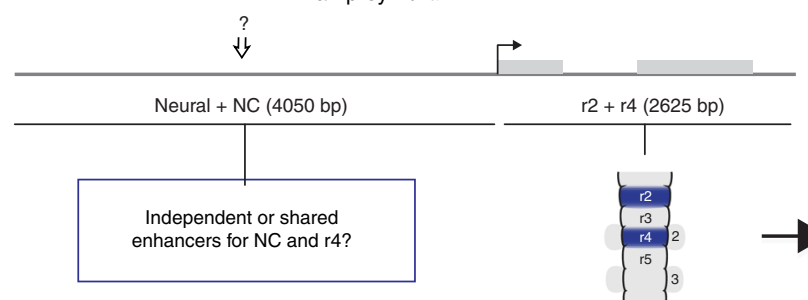
r2 + r4 (2625 bp)
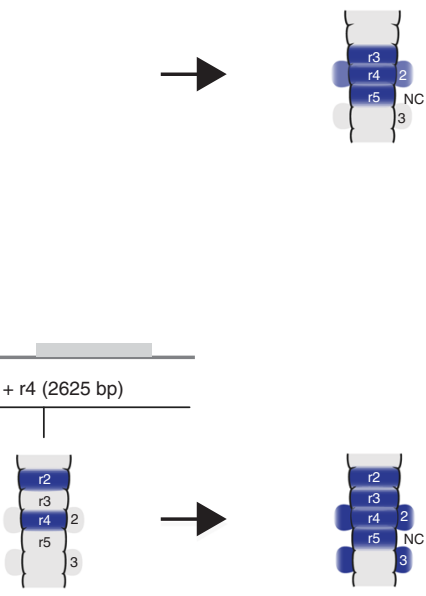

Fig. 1 Characterized Hox2 enhancers regulating neural crest (NC) and rhombomeric expression from mouse and lamprey. Schematic diagrams depicting the known enhancers regulating mouse Hoxa2 (a) and Hoxb2 (b), and lamprey hox 2 ( $\mathbf{c}$ ), in rhombomeres ( $r$ ) and NC. For each locus, the gene exons are represented by grey boxes and the transcriptional start site by an arrow. Enhancers are marked as black lines below the loci, with their activity domains illustrated by blue shading in schematic dorsal views of the hindbrain ( $2-5)$ and pharyngeal arches (2-3). For the mouse loci, characterised cis-elements contributing to enhancer function are depicted as coloured boxes: hindbrain elements in purple and NC elements in green (not drawn to scale). Known direct inputs from transcription factors into these cis-elements are depicted by arrows, with unknown inputs shown as question marks. Hoxa2 is regulated in $r 4$ and $\mathrm{r} 4$-derived NC (PA2) by independent enhancers (a). Hoxb2 expression in $\mathrm{r} 4$ and NC is mediated by a single enhancer, through cis-elements bound by Meis and Pbx-Hox factors (b). Since these elements have dual hindbrain/NC activity they are depicted in both purple and green. Genomic regions from the lamprey hox $\alpha 2$ locus have enhancer activity, with an $\mathrm{r} 2 / \mathrm{r} 4$ enhancer positioned within the exons and intron (c). The hox $\alpha 2$-hox $\alpha 3$ intergenic region drives reporter expression in the hindbrain and NC. However, it is not known whether this is through independent or shared NC/hindbrain enhancers, specific cis-elements have not been identified, and the relationship of this region to the gnathostome Hoxa2 and Hoxb2 enhancers is unclear

Gnathostome Hox2 NC enhancers function in lamprey. To explore conservation of the regulatory network upstream of Hox2 in the NC, we sought to perform cross-species enhancer analysis by testing gnathostome NC enhancers for activity in lamprey embryos. As part of this approach, we wanted a way to globally monitor the $\mathrm{NC}$ in vivo. In zebrafish, the crestin promoter/ enhancer element is a highly specific tool for monitoring NC development, as it is active from pre- to post-migratory stages across multiple axial levels, making it a good candidate for marking NC in lamprey ${ }^{26}$. In lamprey transgenic reporter assays, the crestin element mediates spatiotemporal expression in NC similar to its activity in zebrafish, and its activity is sensitive to perturbation of the same transcription factor binding sites (Supplementary Figure. 1a-d; Fig. 3b; Supplementary Table 1).
Initial green fluorescent protein (GFP) expression in premigratory $\mathrm{NC}$ was observed at st 21 and maintained as $\mathrm{NC}$ delaminated and migrated ventro-laterally to populate the pharynx (Supplementary Figure $1 \mathrm{~b}-\mathrm{c}$ ). Frontal sections at st24 revealed that GFP transcripts are present in the NC-derived pharyngeal mesenchyme (Fig. 3d). These data demonstrate that NC cells can be labelled and visualized in vivo by a reporter assay in the developing lamprey and suggest that the crestin element is interpreted in lamprey by conserved upstream components of an ancestral NC GRN. This serves as a proof of concept for interspecies analysis of NC enhancers.

Next, we investigated whether upstream regulatory inputs required for Hoxa2 expression in gnathostome $\mathrm{NC}$ are present in lamprey using homologous Hoxa2 NC enhancers from three 
a
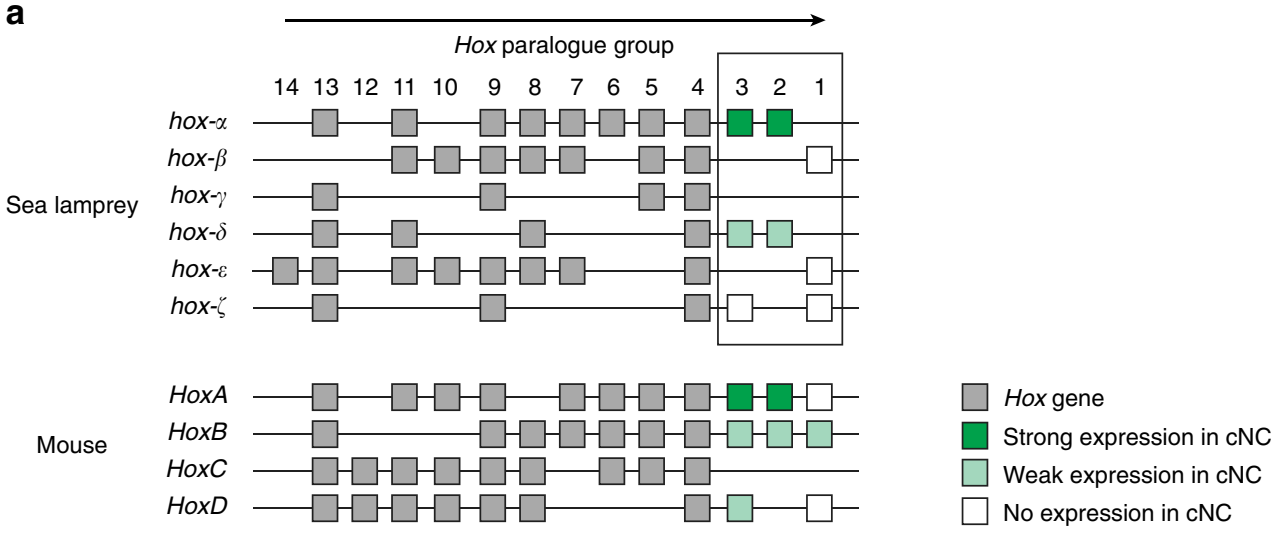

b

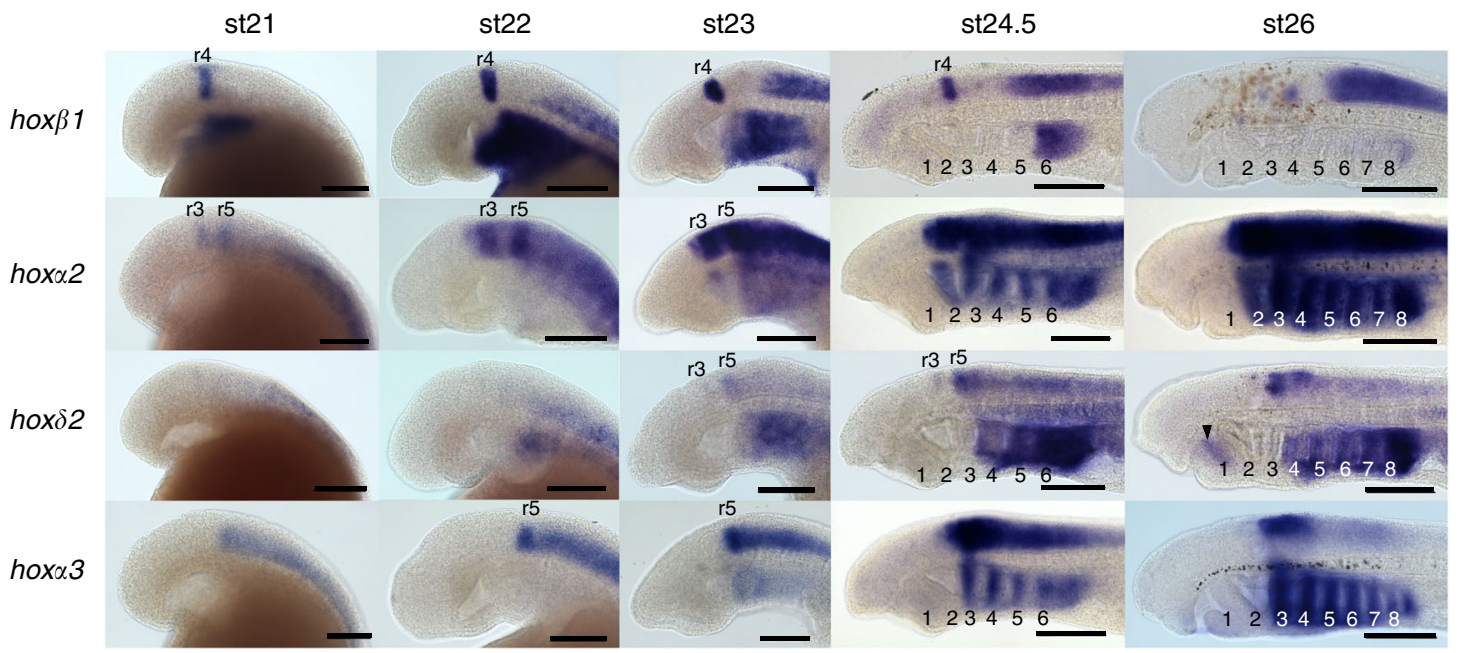

c

$\operatorname{hox} \beta 1$

hox 22
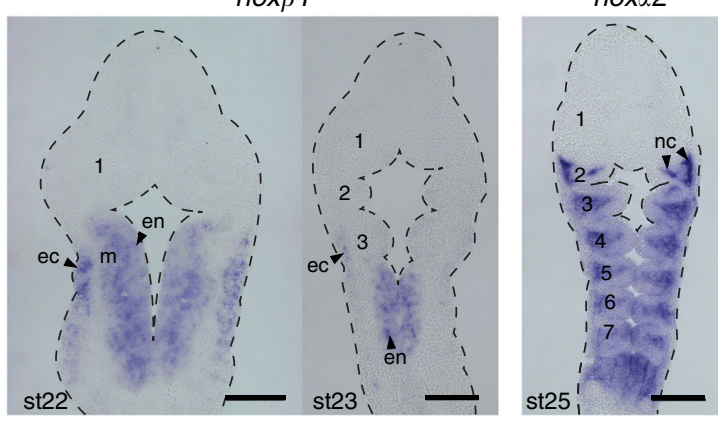

hox $\delta 2$

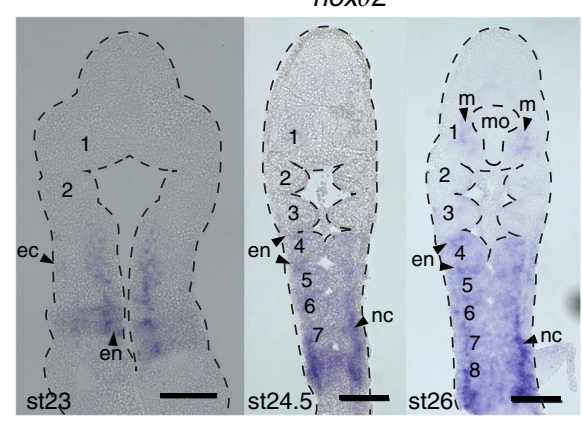

hox 3

d
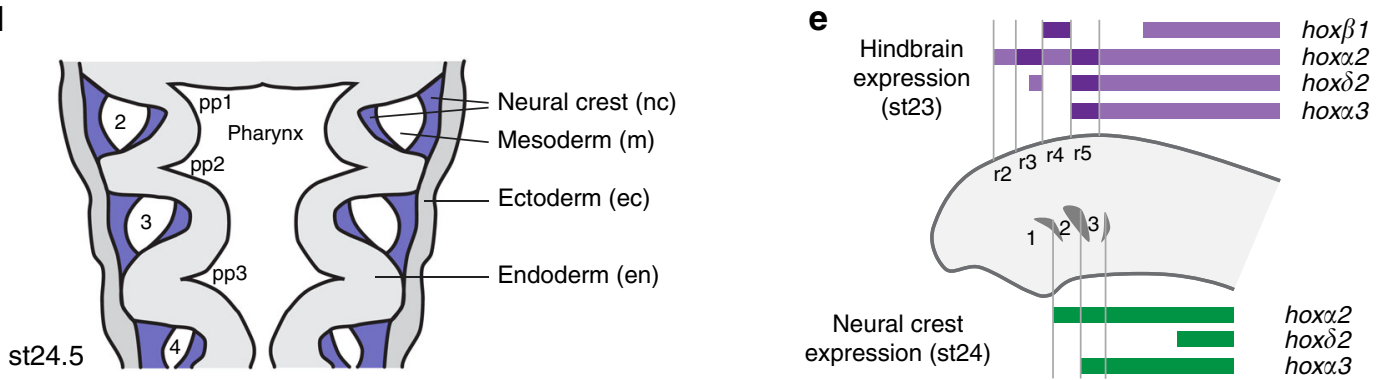

gnathostomes (zebrafish (zf), fugu (f), mouse (m)) (Fig. 3a). In both lamprey and zebrafish embryos, all three gnathostome enhancers mediated GFP reporter expression in the developing pharynx posterior to PA1 (Fig. 3b; Supplementary Table 2; Supplementary Figure 2). Frontal sections of transient transgenic lamprey embryos confirmed that GFP transcripts were

prominently expressed in NC-derived pharyngeal mesenchyme equivalent to crestin reporter expression domains and endogenous hoxa2 (Fig. 3d).

In gnathostomes, $\mathrm{NC}$ Hoxa2 expression is regulated by 5 -flanking elements $(N C 1-5)$ that partially overlap those of a separate $\mathrm{r} 3 / \mathrm{r} 5$ enhancer (RE1-5, Krox20, Sox) (Figs. 1a, 3a) ${ }^{10,27}$. 
Fig. 2 Embryonic time course showing expression of hox genes in the lamprey hindbrain and cranial neural crest (NC). a Genomic organization of Hox genes in lamprey and mouse. Boxes represent Hox genes, which are organized into paralogue groups based on their sequence. The arrow above the clusters denotes the direction of Hox gene transcription. Lamprey hox genes from paralogue groups 1-3 were examined for NC expression in this study and their expression in cranial NC is denoted by green/white shading. b Lateral views of lamprey embryos from stages (st) 21 to 26 , showing hox gene expression domains in the developing head. Pharyngeal arches are numbered and rhombomere-specific domains ( $r$ ) indicated. The arrowhead marks weak hox $\delta 2$ expression in mandibular mesoderm at st26. c Frontal sections through lamprey embryos showing hox gene expression domains within the developing pharynx. Pharyngeal arches are numbered. d Schematic of a frontal section through the lamprey st24.5 embryonic pharynx with tissue domains annotated; NC domains are shaded in blue. Scale bars: $200 \mu \mathrm{m}$ (b); $100 \mu \mathrm{m}$ (c). e Schematic depicting hox expression in the lamprey hindbrain and NC at st23 and st24. ec, ectoderm; en, endoderm; m, mesoderm; mo, mouth; nc, neural crest; r, rhombomere; st, stage

Of these, the NC3 element is the most highly conserved: global sequence alignment using Multi-LAGAN ${ }^{28}$ identified sequence conservation of NC3 extending to sharks (Fig. 3a). In previous work, two 15 bp deletions within NC3 were each found to abolish $\mathrm{NC}$ reporter expression in mouse ${ }^{10}$. To determine whether the same cis-elements are required for $\mathrm{NC}$ activity of $\operatorname{Hoxa2}(\mathrm{m})$ in lamprey, we generated two variants with these deletions in NC3. While hindbrain activity persisted, these reporters exhibited severely diminished NC activity in zebrafish and lamprey (Fig. 3a, c; Supplementary Figure 3a, b; Supplementary Table 3). Analogously, activity of a gnathostome Hoxb2 NC enhancer (hoxb2a $(z f))$ also depended upon the same regulatory sites in lamprey as in zebrafish (Supplementary Figure 3c, d). Since Hoxa2( $m$ ) and hoxb2a $(z f) \mathrm{NC}$ enhancers require the same cis-motifs across distant species, this suggests that an ancestral GRN upstream of Hox2 in NC patterning has been retained in lamprey and gnathostome lineages.

Conservation of binding sites in a lamprey hoxa 2 NC enhancer. Gnathostome Hoxa2 and lamprey hoxa 2 share similarity in their cis-regulatory architecture for rhombomeric hindbrain expression, as $\mathrm{r} 2$ and $\mathrm{r} 4$ enhancers are embedded in conserved locations (Fig. 1a, c) ${ }^{13,18}$. However, our global sequence alignment failed to reveal conservation of NC regulatory elements (NC1-5) 5' of the lamprey hox2 genes (Fig. 3a). Hence, we manually searched for short sequence motifs within an enhancer (hox $2-4 \mathrm{~kb}$ ) in the $5^{\prime}$-flanking region of hox 2 that recapitulates endogenous hoxa2 expression in the hindbrain (r3-r5), NC posterior to PA1 and somites (Fig. 4a-e; Supplementary Figure 4; Supplementary Table 2). In mouse Hoxa2, the most highly conserved elements required for $\mathrm{NC}$ expression are $\mathrm{NC} 3$ and part of $N C 2$, while those necessary for $\mathrm{r} 3 / \mathrm{r} 5$ activity are Krox20, Sox, RE2, and $R E 3$ sites. Surveying the lamprey enhancer, we identified short matching sequences for Krox20, Sox, and NC3 (Fig. 4f; Supplementary Figure 5). To address whether a smaller region containing these sites retains enhancer activity, we cloned a 1530 bp region encompassing the conserved sites with $\sim 500$ bp on each side (hox 2 element $A$ ) and demonstrated that it mediates reporter expression equivalent to hox $2-4 k b$ in lamprey (Fig. $4 \mathrm{~g}, \mathrm{j}$ ). To test if the conserved sites are required for tissue-specific enhancer activity, we assayed variants of hox $2-4 k b$ in which these sites were mutated (Fig. 4f, h-j). Deleting Krox20-Sox sites ( $\triangle$ Krox20) resulted in the loss of $\mathrm{r} 3 / \mathrm{r} 5$ expression, but maintenance in $\mathrm{r} 4$ and NC (Fig. 4f, h). In contrast, deleting the conserved sites within NC3 $(\triangle N C 3)$ caused loss of all rhombomeric and NC activities, but somitic expression is retained (Fig. $4 \mathrm{f}, \mathrm{i}-\mathrm{j}$ ).

Inspection of the conserved sites between the lamprey hox $\alpha 2$ and gnathostome Hoxa2 enhancers revealed that they match closely to consensus transcription factor binding site motifs for factors involved in early hindbrain and NC patterning. In addition to the previously characterised Krox20 sites, we identified three short blocks of conserved sequence that correspond to consensus binding motifs for Sox, Meis, and Pbx-Hox factors (Fig. 4f). These motifs each fall within regions functionally required for enhancer activity in gnathostomes and lamprey, notably NC3 (Fig. 4a, f, h-j, Supplementary Figure $3 \mathrm{a}-\mathrm{b}$ ), suggesting that Meis, Pbx, and Hox factors may provide conserved and essential inputs into these enhancers. Thus, lamprey hoxa2 and gnathostome Hoxa2 appear to be regulated in the hindbrain and NC through conserved transcription factor binding sites retained during vertebrate evolution. This provides further support for an ancestral GRN upstream of Hox2 in NC patterning that has been retained in lamprey and gnathostomes.

Hoxa2 and Hoxb2 NC enhancers are divergent paralogues. The identification of conserved Meis, Pbx, and Hox binding motifs in the lamprey hox 2 and gnathostome Hoxa2 enhancers is significant as equivalent sites are present and functionally required in the mouse Hoxb2 and zebrafish hoxb2a enhancers (Fig. 1b; Supplementary Figure $3 \mathrm{c}$ ). To explore whether these are homologous sites and to interrogate common and diverged features of the hoxa2, Hoxa2, and Hoxb2 enhancers, we used the Krox20 sites, implicated in $\mathrm{r} 3 / \mathrm{r} 5$ expression, as an anchor to align the enhancer sequences. This revealed striking conservation of the sequence and order of Krox20, Sox, Meis, and Pbx-Hox sites between these enhancers, with relatively low sequence conservation within the intervening regions (Fig. 5a; Supplementary Figure 6). Based on these conserved sites and relative positions, we infer that Hoxa2 and Hoxb2 NC enhancers are ancient paralogues, derived from an ancestral vertebrate Hox2 enhancer that contained these sites. These paralogous enhancers appear to have diverged in gnathostomes, such that the mouse Hoxa 2 enhancer is inactive in $\mathrm{r} 4$ but expressed in $\mathrm{r} 4$-derived $\mathrm{NC}$, while the Hoxb2 enhancer is active in both $\mathrm{r} 4$ and its NC (Fig. 5a) ${ }^{11}$. The lamprey hox 2 NC enhancer exhibits the combined activity of both mouse Hoxa2 and Hoxb2 enhancers, suggesting that it may reflect the ancestral state. We searched upstream of lamprey hox $\delta 2$, finding conservation of Krox 20 and Sox sites, but no Meis or $\mathrm{Pbx}-\mathrm{Hox}$ sites (Fig. 5b). This suggests that the ancestral sites for $\mathrm{r} 4 / \mathrm{NC}$ enhancer activity were lost upstream of hox $\delta 2$, consistent with the expression of hox $\delta 2$ in $\mathrm{r} 3 / \mathrm{r} 5$ but not in $\mathrm{r} 4$ and $\mathrm{r} 4$-derived NC (Fig. 2b, c).

Lamprey meisC and hoxa 2 are similarly expressed in NC. Identification of Meis and Pbx-Hox consensus binding motifs in the hox 2 enhancers of lamprey and gnathostomes implies that these factors may play conserved roles in regulating vertebrate Hox2 genes in the hindbrain and NC. Meis, Prep, and Pbx are members of the TALE (Three-Amino-Acid-Loop-Extension) homeodomain family ${ }^{29}$ that have diverse roles in patterning tissues, including hindbrain and $\mathrm{NC}^{30-33}$. Additionally, they can act as cofactors for other transcription factors, including Hox proteins ${ }^{34}$. To initially explore whether Meis factors are linked with regulation of $\mathrm{NC}$ in lamprey, we characterized expression of four lamprey meis genes. We found that meis $C$ shows early $\mathrm{NC}$ expression with a spatiotemporal pattern similar to hox 2 
a

Hindbrain element

Neural crest element
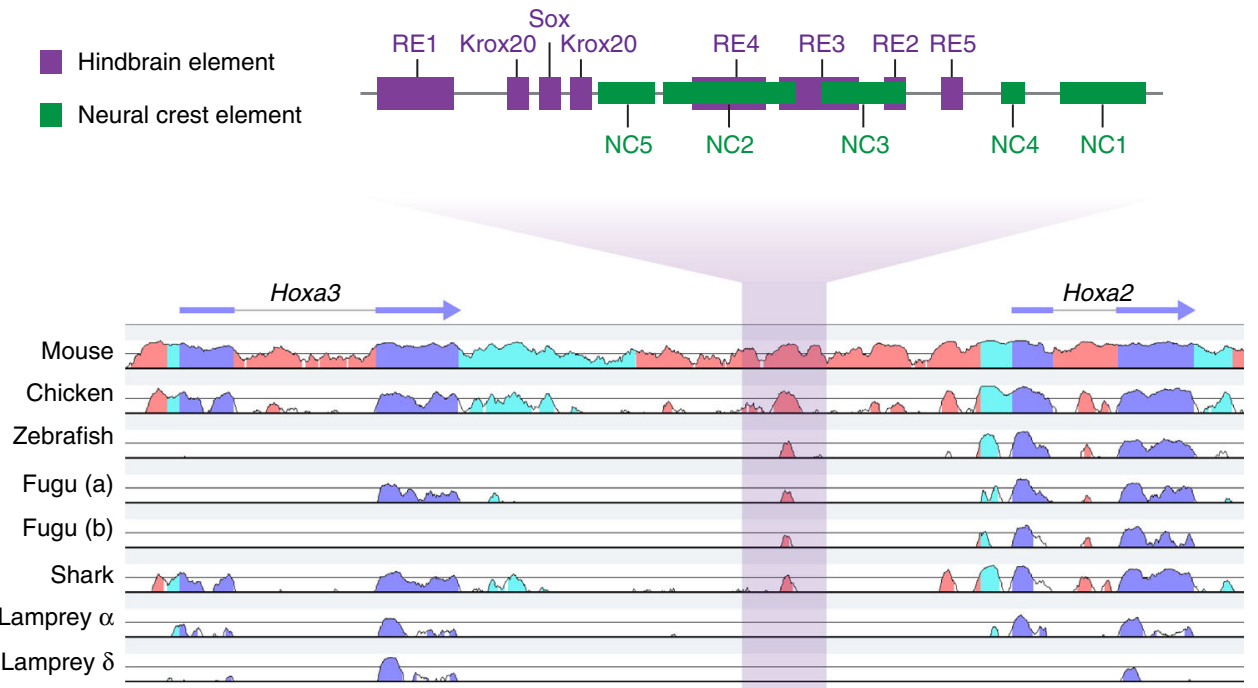

$1 \mathrm{~kb}$

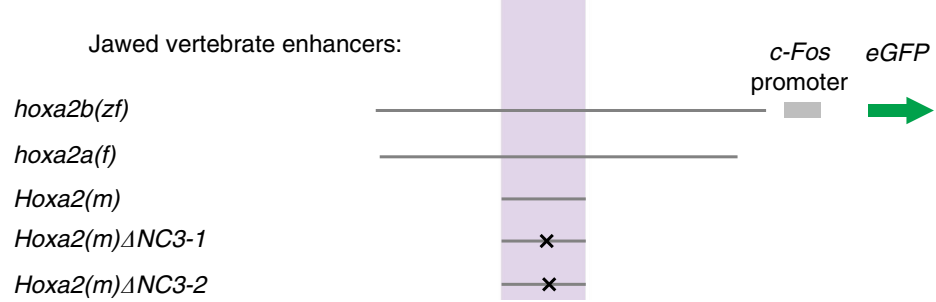

b

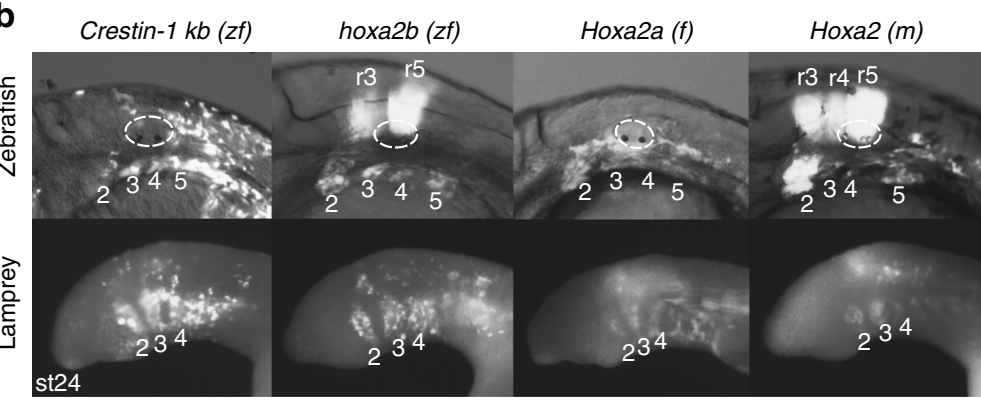

C $_{\text {Hoxa2(m) } \triangle N C 3-1} \operatorname{Hoxa2}(m) \triangle N C 3-2$

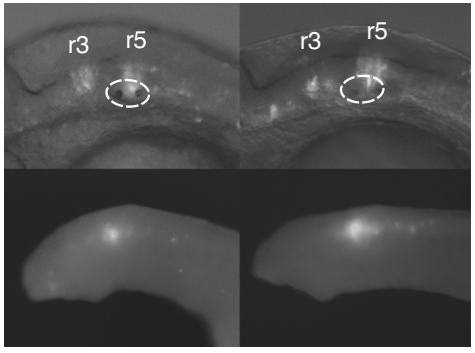

d
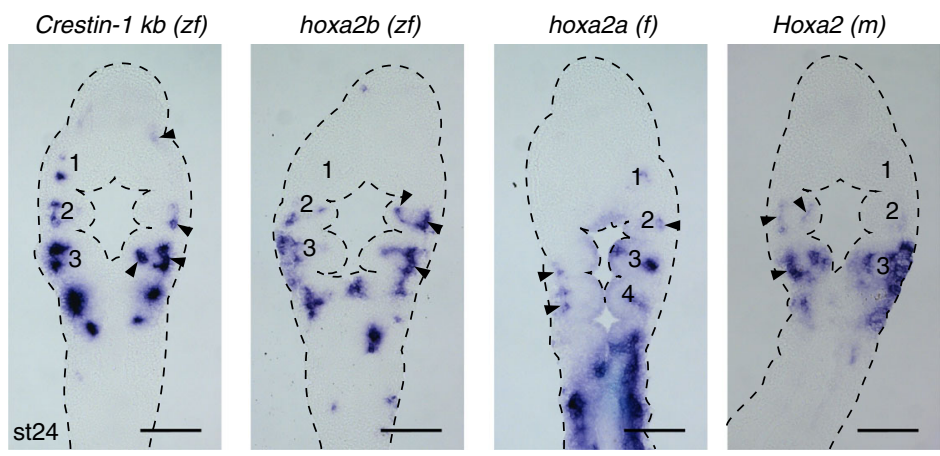

Fig. 3 Conserved activity of gnathostome Hoxa2 neural crest (NC) enhancers in zebrafish and lamprey. a Sequence alignment of gnathostome Hoxa2-Hoxa3 and lamprey hox2-hox3 gene loci against the human locus. Conserved non-coding sequences (pink), untranslated regions (UTRs) (cyan) and coding sequences (blue) are highlighted. The relative locations of the mouse hindbrain and NC cis-elements (top) are shown. Gnathostome Hoxa2 enhancers used for cross-species reporter analysis are detailed below the alignment. Letters within parenthesis indicate species of origin of the enhancer: $z f$, zebrafish; $f$, fugu; $m$, mouse. b, c Green fluorescent protein (GFP) reporter expression in zebrafish and lamprey embryos (lateral views), mediated by wild-type (b) and mutated (c) gnathostome NC enhancers. For zebrafish, the otic vesicle is circled and GFP expression in rhombomeres ( $r$ ) and pharyngeal arches (2-5) indicated. Lamprey pharyngeal arches are labelled (2-4). GFP-expressing embryos shown are representative of the expression potential of the reporter construct in each case, as inferred from screening many (typically more than 100) injected embryos. Supplementary Table 2 provides the number of embryos and details of specific expression for all constructs in lamprey. Injection statistics for the transient transgenic zebrafish embryos shown in c are given in Supplementary Table 3. d Frontal sections through the transient transgenic lamprey embryos shown in Fig. 3b, with GFP transcripts detected by in situ hybridisation, revealing expression in NC-derived mesenchyme (arrowheads) in the pharyngeal arches (numbered). Scale bars: $100 \mu \mathrm{m}$ 
a
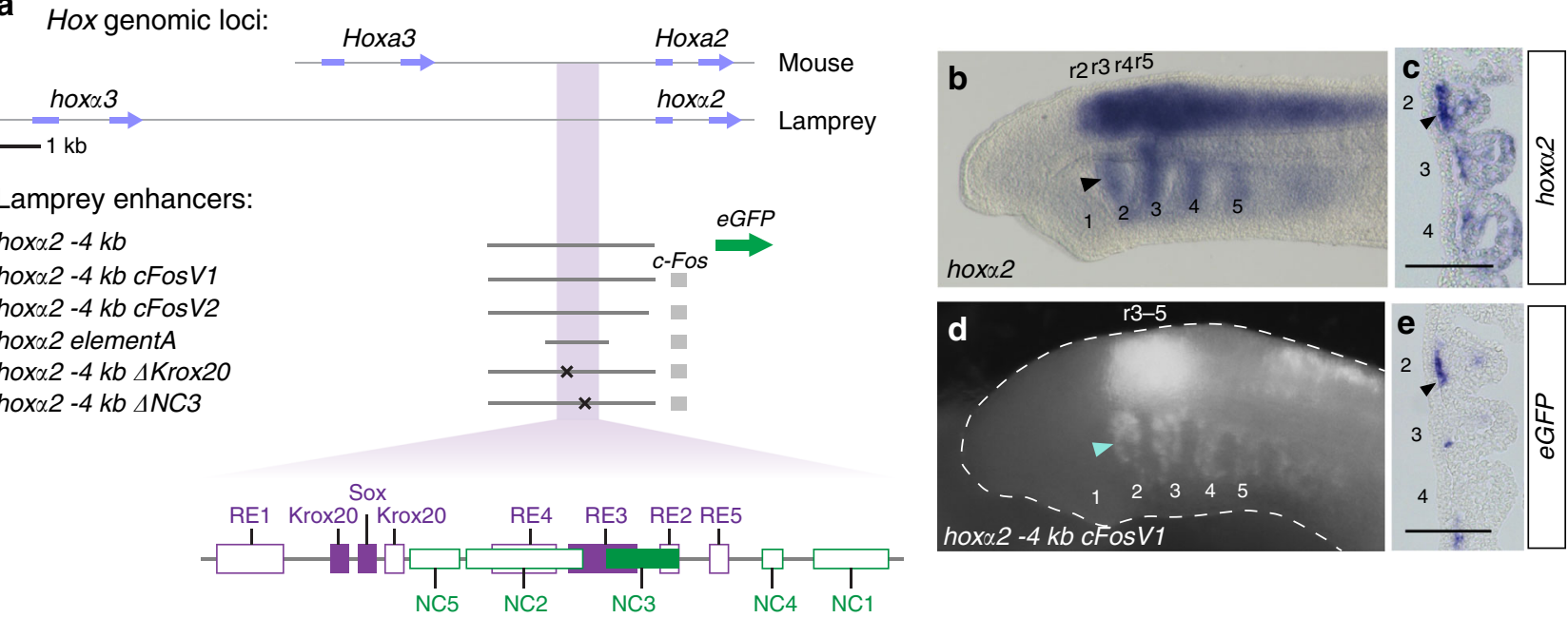

f

\section{Krox20}

\section{Sox}
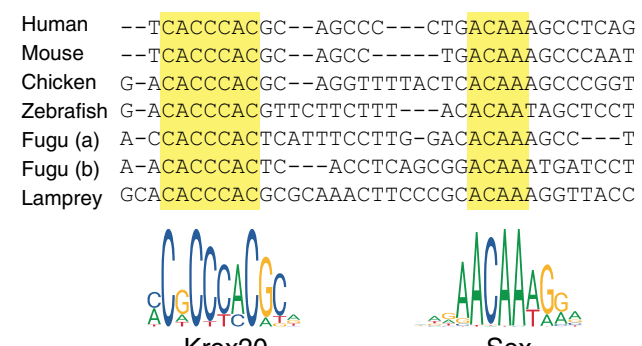

Krox20

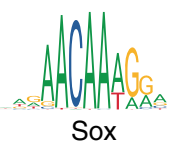

$\Delta K r o x 20$

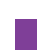

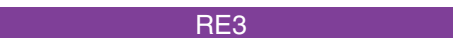
NC3

CTIAAGGGCTAGAAGCTGTCAAGGCTTTT--GGTGAGCAAGATTGATCGCGCCCAGACT

250-400bp interval of non-aligning sequence

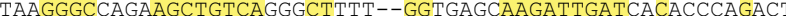
CTTAAGGGCCAGAAGCTGTCAAACCTTTT--GGCGGCCAAGATTGATCGCGCGCAGACA CTTA-GGGCACTAAGCTGTCAGACCTTTT--GGCGTGTAAGATTGATAGCGTGCAGGCT GCGA-GGGCACCGAGCTGTCAGACCTTTT--GGCGAGTAAGATTGATCGCGCACAGGCT CTTA-GGGCAGGGAGCTGTCAGACCTTTT--GGCGTGAAAGATTGATCACACTCAGGGA GTGCAGGGCAAG-AGCTGTCAAGGCTCGCCCGGCCGGGAAGATTGATAGCGAGG-GGCT
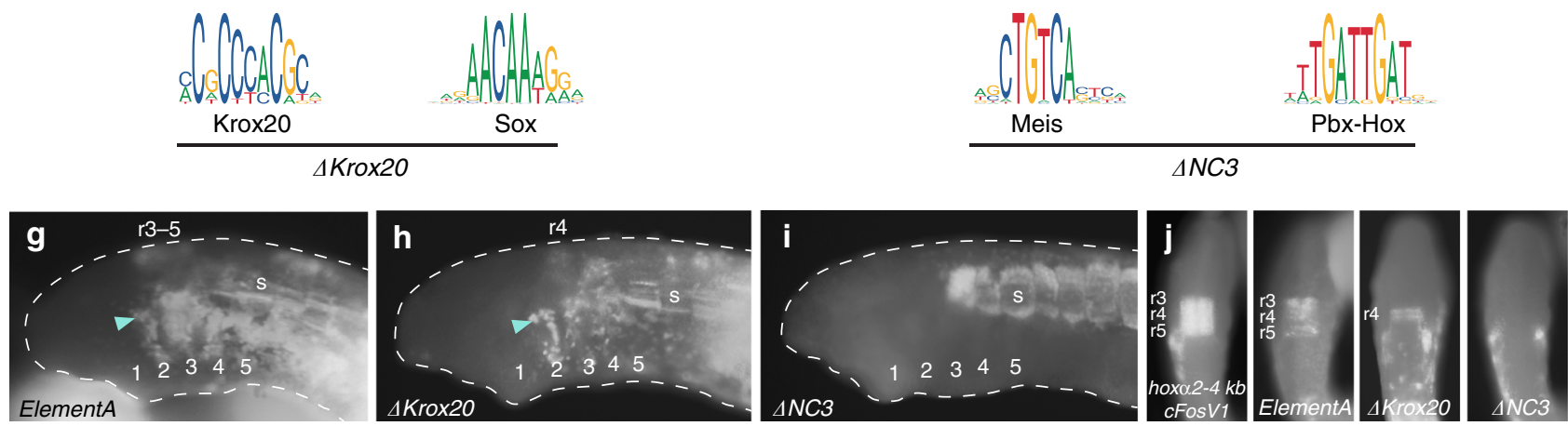

Fig. 4 Characterization of a lamprey hox $\alpha 2$ neural crest (NC)/hindbrain enhancer. a The mouse Hoxa2-Hoxa3 genomic region and its equivalent from the lamprey hox $\alpha$ cluster are shown, with Hox gene exons annotated (blue arrows). hox $\alpha 2$ upstream regions assayed for reporter activity in this study, with or without the c-Fos minimal promoter, are shown. b-e Lateral views (b, d) and frontal sections (c, e) of st24.5 lamprey embryos, comparing endogenous expression of hox $2(\mathbf{b}, \mathbf{c})$ to GFP reporter expression mediated by hox $\alpha 2-4 \mathrm{~kb}(\mathbf{d}, \mathbf{e})$. Pharyngeal arches are numbered and rhombomeric expression detailed. Arrowheads point to PA2 NC expression. f Multiple sequence alignment of the Hoxa2 NC enhancer from gnathostomes with the lamprey hox 22 enhancer, showing conserved sites (yellow). The positions of characterized mouse cis-elements (Krox20, Sox, RE2-3, NC3) are marked above the alignment. The enhancer schematic (a) shows the position of these elements within the assayed hox $\alpha 2$ upstream regions, with conserved (shaded boxes) or divergent (empty boxes) cis-elements highlighted. Consensus binding motifs from the JASPAR database ${ }^{76}$ for Krox $20^{77}$, Sox $11^{78}$, Meis $1^{79}$, and Pbx-Hox ${ }^{80}$ factors are shown below the alignment, as well as sequences deleted in hox $\alpha 2-4 \mathrm{~kb} \Delta \mathrm{Krox} 20$ and $\Delta N C 3$ variants. The non-aligning interval between these conserved regions is $\sim 250-400$ bp and varies in length between species. Supplementary Figure 5 contains the full alignment. $\mathbf{g}$-j Lateral ( $\mathbf{g}$-i) and dorsal (j) views of st24.5 lamprey embryos showing GFP reporter expression driven by the enhancers detailed in a. Pharyngeal arches are numbered, with expression in rhombomeres ( $r$ ) and somites ( $s$ ) annotated. GFP-expressing embryos shown are representative of the expression potential of the reporter construct in each case, as inferred from screening many (typically more than 100) injected embryos. Supplementary Table 2 provides the number of embryos and details of specific expression for all constructs in lamprey

(Fig. 6a-d). This expression data and the conserved consensus Meis binding sites are consistent with the notion that meis genes may have played an ancestral role in regulating Hox2 in NC during vertebrate evolution.

Occupancy of TALE and Hox proteins on Hox2 enhancers. Hoxb1, Pbx, and Meis bind to sites within the mouse Hoxb2 hindbrain/NC enhancer that are required for its activity ${ }^{11,17}$. The presence of homologous sites within NC3 of the mouse Hoxa2 enhancer suggests that such factors may also regulate its activity in the hindbrain and NC. This is significant because previous characterisation of this $\mathrm{NC}$ enhancer did not uncover factors that bind to NC3 nor provide insight into its underlying regulatory mechanism ${ }^{10,35}$. The divergent activities of the Hoxa2 and Hoxb2 enhancers, particularly in $\mathrm{r} 4$, suggest that there may be differences in their interactions with upstream regulatory factors despite the presence of homologous binding sites. This led us to investigate binding properties of Meis, $\mathrm{Pbx}$, and Hox proteins on these enhancers. 
a

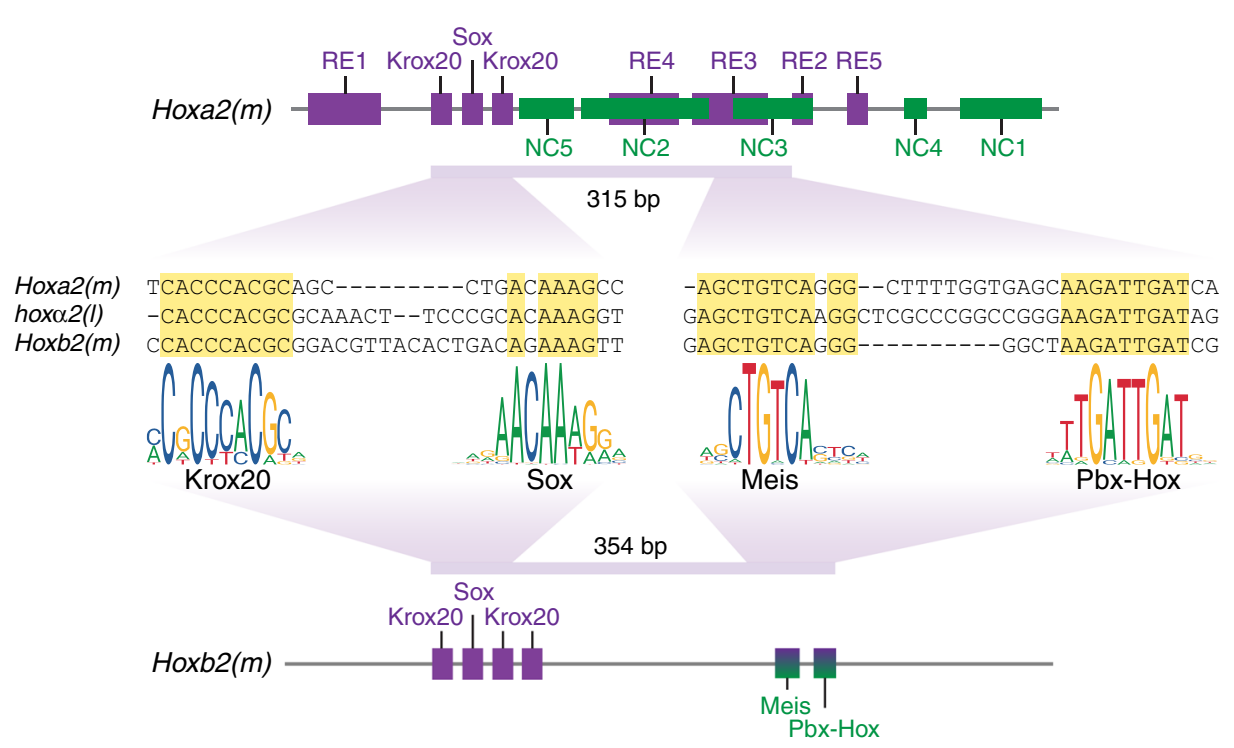

NC enhancer activity
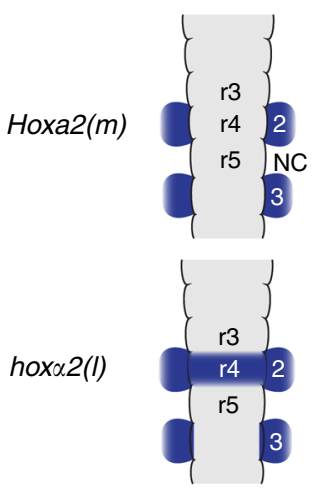

$\operatorname{Hoxb2}(m)\left\{\begin{array}{c} \\ r 3 \\ r 4 \\ r 5 \\ r\end{array}\right.$

b Lamprey Hox genomic loci

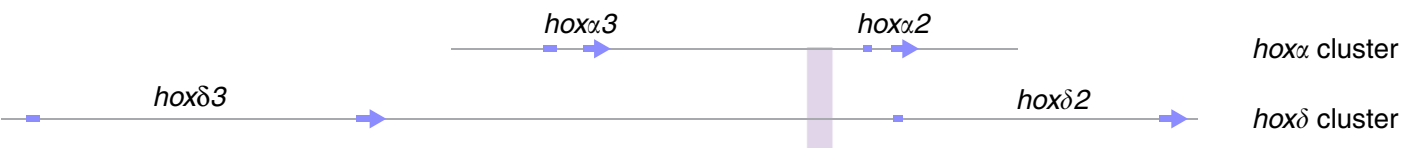

$\underline{2 \mathrm{~kb}}$

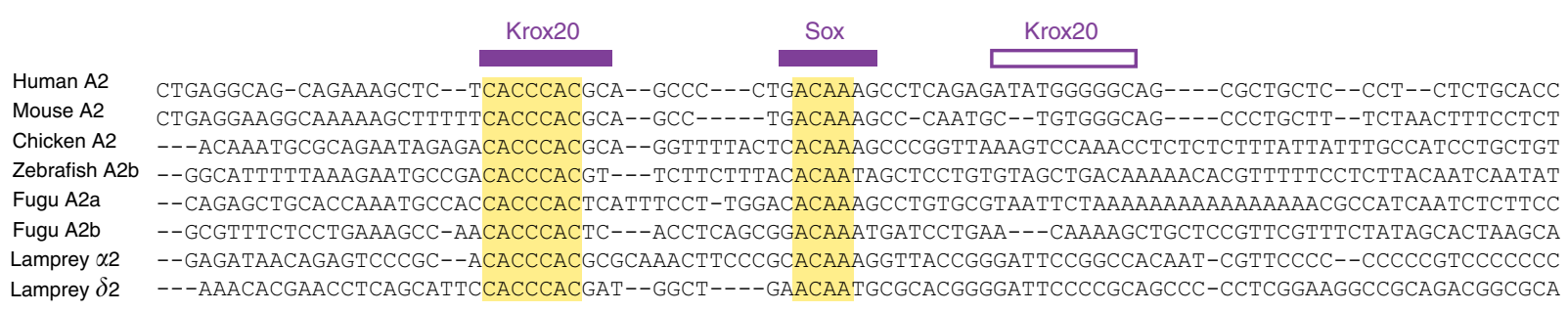

Fig. 5 Hoxa2 and Hoxb2 neural crest (NC) enhancers are ancient paralogues and the lamprey hox 2 enhancer appears to reflect the ancestral state. a Sequence alignment of mouse (m) Hoxa2 and Hoxb2 NC enhancers with that of lamprey (I) hox 2 , revealing short conserved sequence blocks (yellow). Corresponding consensus binding motifs for Krox20, Sox, Meis, and Pbx-Hox factors are shown below the alignment. These conserved sequences map to characterized cis-elements required for hindbrain (purple) or NC (green) activity in the mouse Hoxa2 (above) and Hoxb2 (below) enhancers. The 315 and $354 \mathrm{bp}$ refer to the precise distances between the $5^{\prime}$ end of the Krox2O site and the $3^{\prime}$ end of the Pbx-Hox site of the mouse Hoxa2 and Hoxb2 enhancers, respectively. The activity of each NC enhancer in the hindbrain and NC is shown in schematic dorsal views. This activity differs between each enhancer, with hoxa2(I) showing the combined output of $\operatorname{Hoxa2}(\mathrm{m})$ and $\operatorname{Hoxb2}(\mathrm{m})$. b Multiple sequence alignment of gnathostome Hoxa2 NC enhancers with a homologous region upstream of lamprey hox $\delta 2$. The lamprey hox $\alpha 2$-hox $\alpha 3$ and hox $\delta 2$-hox $\delta 3$ genomic loci are depicted, with hox gene exons annotated (blue arrows). The multiple sequence alignment reveals conservation of a Krox 20 and a Sox site upstream of hox $\delta 2$ (yellow shading in alignment), but other cis-elements, including NC3, are not conserved in sequence. This is depicted in the enhancer schematic, which details the conserved (shaded boxes) and divergent (empty boxes) cis-elements upstream of hox $\delta 2$

For insight into NC, we harnessed published genome-wide binding data for Meis, $\mathrm{Pbx}$, and Hoxa2 in PA2 of mouse embryos $^{36,37}$. Focusing on the NC enhancers of Hoxa2 (NC3) and Hoxb2 (HRE), we observed similar binding profiles for these factors over each of the enhancers, consistent with their regulatory activity in NC. There is enrichment for occupancy of Meis and $\mathrm{Pbx}$, in keeping with a role for these TALE factors in controlling Hox2 NC activity (Fig. 7a, b). It is interesting that Hoxa2 also binds to these enhancers in PA2, suggesting that there may be auto- and cross-regulatory inputs from Hox 2 proteins into the NC Hox code. This is analogous to the important roles for auto- and cross-regulatory circuits in regulating Hox expression in other tissues, including hindbrain rhombomeres ${ }^{11,13,15}$. Since $\mathrm{Pbx}$ and Meis can act as Hox cofactors $^{38}$, they may interact with Hoxa2 on these NC enhancers. Together with the presence of essential Meis motifs 

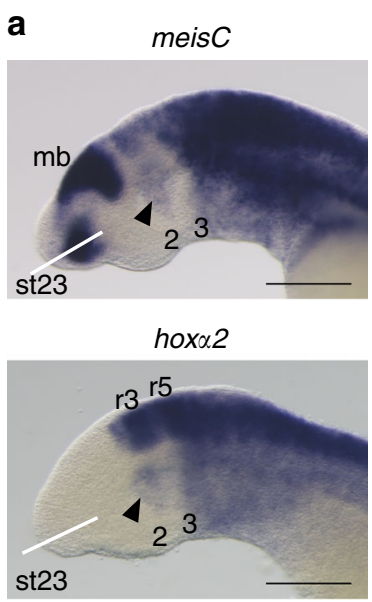

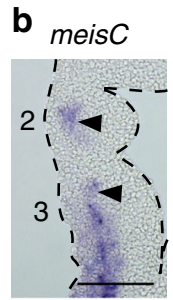

hox $\alpha 2$

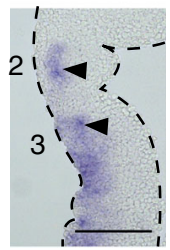

c

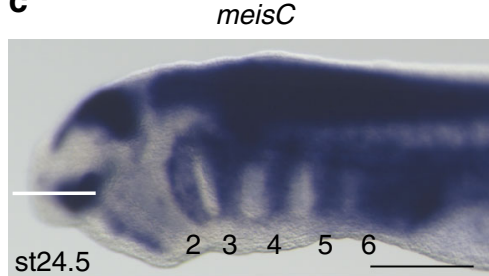

hox 22

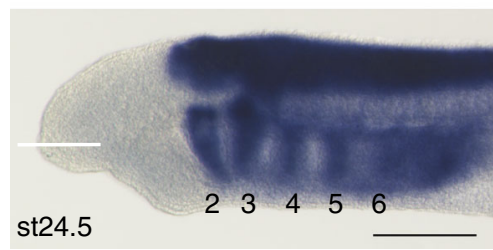

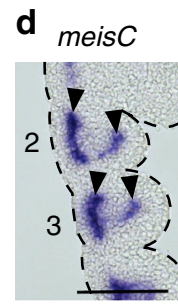

hox $\alpha 2$

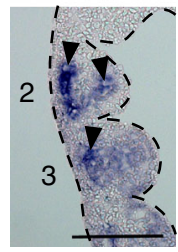

Fig. 6 Endogenous expression of meis $C$ in neural crest (NC) overlaps with that of hox 22 in lamprey embryos. a Lateral views (a, c) and frontal sections (b, d) are shown for embryos at st23 (a, b) and st24.5 (c, d). White lines in $\mathbf{a}$ and $\mathbf{c}$ denote planes of sections in $\mathbf{b}$ and $\mathbf{d}$. Pharyngeal arches are numbered, arrows denote expression in NC. Scale bars: $200 \mu \mathrm{m}(\mathbf{a}, \mathbf{c}) ; 100 \mu \mathrm{m}(\mathbf{b}, \mathbf{d})$. mb, mid-brain

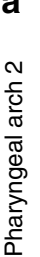

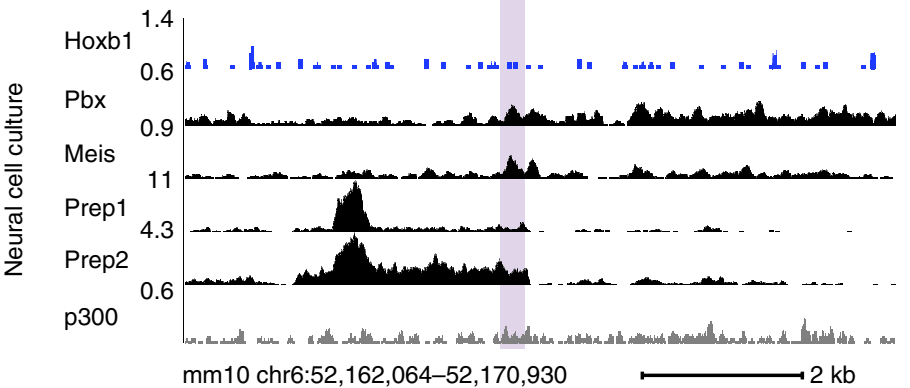

b

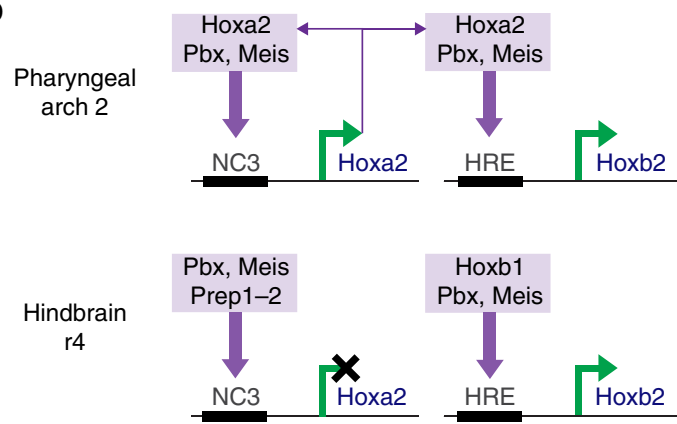

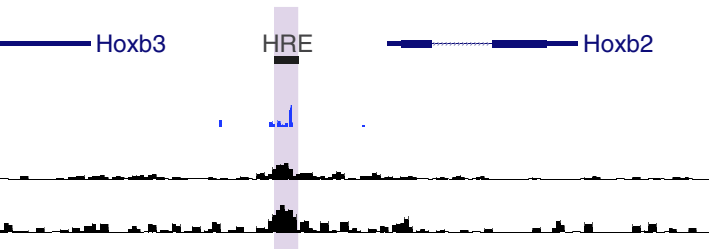

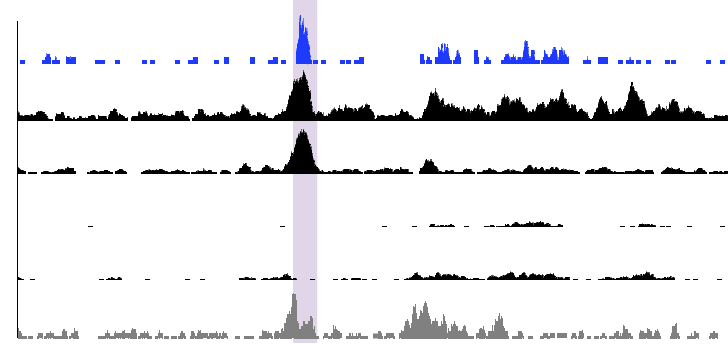

mm10 chr11: 96,346,792-96,355,658

Fig. 7 Hoxa2 and Hoxb2 enhancers exhibit differential TALE (Three-Amino-Acid-Loop-Extension) and Hox binding correlating with their tissue-specific activities. a DNA-binding profiles for Hox, TALE, and p300 factors in neural cell culture and pharyngeal arch 2 tissues at the mouse Hoxa2 (NC3) and Hoxb2 (HRE) neural crest (NC) enhancers (highlighted in purple). Genes are annotated (top) and are transcribed from left-to-right. b Summary diagram of characterized differential regulatory inputs (purple arrows) from Hox and TALE factors (inferred from a) into the mouse Hoxa2 and Hoxb2 NC enhancers in pharyngeal arch 2 (NC) and hindbrain r4 in vivo. Activation or inactivation of transcription is depicted by green arrows or a black cross, respectively. Purple arrows from the Hoxa2 gene indicate auto-/cross-regulation

and bipartite $\mathrm{Pbx}-\mathrm{Hox}$ sites, this raises the possibility of both Hox-dependent and -independent inputs of $\mathrm{Pbx}$ and Meis into NC Hox expression.

With respect to the developing hindbrain, chromatin immunoprecipitation-sequencing (ChIP-seq) approaches are not feasible for individual rhombomeres due to the small number of cells and limiting amounts of embryonic material. In addition, there is no suitable anti-Hoxb1 antibody for ChIP-seq experiments. To circumvent these challenges, we generated a mouse embryonic stem (ES) cell line (KH2) carrying an inducible 
locus-specific insertion of Hoxb1 marked with Flag epitopes and used it in combination with programmed differentiation of ES cells into neural fates. This enabled us to use anti-Flag antibodies for Hoxb1 ChIP-seq and to obtain sufficient cell populations for a comparative series of genome-wide binding experiments. This cell culture system has previously been shown to exhibit global changes of gene expression that are similar to early in vivo phases of neural development, including the sequential activation of hindbrain-expressed Hox genes and their cofactors (such as Meis 2$)^{39,40}$. We have previously applied this system to investigate the genome-wide binding properties of Hoxal and TALE proteins in neural cells, uncovering in vivo regulatory interactions relevant to hindbrain patterning $39-42$.

Using this same approach for neural cells, we found similarities and significant differences in binding patterns of Hox and TALE proteins between the paralogous Hox2 enhancers (Fig. 7a, b). The Hoxb2 (HRE) enhancer shows robust binding of Hoxb1, Pbx, and Meis, plus prominent p300 recruitment, consistent with their established in vivo role in mediating Hoxb2 expression in $\mathrm{r}^{11,17}$. In contrast, the Hoxa2 (NC3) enhancer lacks discernable binding of Hoxb1, has reduced levels of Pbx and Meis occupancy, and displays differential binding patterns of Prep1 and Prep2. These properties, in combination with absence of p300, directly correlate with its lack of activity in $\mathrm{r} 4$. These differences in TALE and Hoxb1 binding and $\mathrm{r} 4$ activity presumably reflect sequence divergence or differences in epigenetic states between the two enhancers. Studies on Pbx-Hox protein binding have shown that small sequence variations within the canonical PbxHox bipartite binding sites influence the selectivity for specific Hox proteins ${ }^{38}$. However, sequence comparisons of the Hoxb2, Hoxa2, and hoxa2 enhancers show that the core consensus Meis and Pbx-Hox sites are identical (Figs. 4f, 5a; Supplementary Figure 6). In contrast, the sequences around these sites differ considerably between enhancers: for example, sequences immediately $5^{\prime}$ of the Meis site are shared between Hoxb2 and hox 2 but not Hoxa2 (Supplementary Figure 6). While these differences in neighbouring sequences may have arisen by sequence drift and be functionally neutral, an intriguing alternative is that they may have functional significance in modulating binding to the conserved motifs and impacting r4 activity. Taken together, ChIP-seq data link TALE (Pbx and Meis) and Hox proteins to regulation of Hox2 genes in both the hindbrain and NC.

\section{Discussion}

Here, we have investigated the ancestral regulation of Hox2 genes in the NC and hindbrain of vertebrates, using interspecies cisregulatory comparisons between gnathostomes and lamprey. We demonstrated that gnathostome Hoxa2 and Hoxb2 NC enhancers are capable of driving equivalent $\mathrm{NC}$ expression in lamprey as they do in gnathostomes and that a homologous enhancer is present upstream of lamprey hox 2 . Sequence comparisons and regulatory analysis revealed conserved Meis, $\mathrm{Pbx}$, and Hox binding sites between gnathostome Hoxa2/Hoxb2 and lamprey hoxa $2 \mathrm{NC}$ enhancers that are required for their activity. The lamprey hox $2 \mathrm{NC}$ enhancer appears to have retained ancestral activity in both $\mathrm{NC}$ and hindbrain, while the paralogous Hoxa2 and Hoxb2 NC enhancers have differentially partitioned NC and hindbrain activities in the gnathostome lineage. Regulatory divergence has also occurred between lamprey Hox2 paralogues, with hox $\delta 2$ appearing to have lost the regulatory sites for $\mathrm{r} 4 / \mathrm{NC}$ enhancer activity. This suggests that a regulatory circuit with input from TALE and Hox proteins was an important component of the GRN for Hox2-dependent NC patterning in ancestral vertebrates that has been maintained during evolution (Fig. 8a). TALE factors are part of an ancient patterning system ${ }^{38,43}$ that may have multiple roles in coupling Hox expression to the core NC GRN. These findings raise a number of interesting points and avenues for further investigation.

At the mechanistic level, little is known with respect to shared versus independent inputs that govern axial patterning in the hindbrain and NC. This is because the mechanisms regulating Hox expression in the NC are relatively unclear compared to the current knowledge of rhombomeric Hox regulation ${ }^{4}$. Analyses of mouse Hoxa2 and Hoxb2 NC enhancers provided conflicting mechanisms for NC expression of Hox genes. Hoxa2 supported evidence for independent enhancers mediating expression in $\mathrm{r} 4$ and r4-derived $\mathrm{NC}$, since the $\mathrm{NC}$ enhancer is not active in $\mathrm{r} 4$ and a separate intronic/exonic enhancer drives $r 4$ expression ${ }^{10,15}$. In contrast, Hoxb2 uses common elements to control $\mathrm{r} 4$ and NC expression, suggesting similar or shared regulatory requirements in these tissues ${ }^{11}$. Our analyses resolve this paradox, providing evidence that the Hoxa2/Hoxb2 NC enhancers each retain conserved Meis, Pbx, and Hox binding sites, which are deployed in slightly different ways in mediating tissue-specific activities. hox 2 is the only lamprey hox 2 paralogue expressed in PA2 NC (Fig. 2b, c) and we uncovered the presence of homologous functional motifs (Meis, Pbx, and Hox) in an upstream enhancer with activity in $\mathrm{r} 4$ and NC. Based on sequence conservation, position, and regulatory activity, we infer that this enhancer is homologous to gnathostome Hoxa2/Hoxb2 NC enhancers. Sequence comparisons suggest that lamprey hox $\delta 2$ has diverged and is missing these NC motifs but retains Krox20 and Sox sites, consistent with its expression in $\mathrm{r} 3 / \mathrm{r} 5$. Since the lamprey hox $\alpha 2$ NC enhancer exhibits the combined activity of the Hoxa2 and Hoxb2 enhancers, we consider it likely that it reflects the ancestral state. Thus, we suggest that Hox2 was ancestrally regulated in $\mathrm{r} 4$ and NC by a shared enhancer through inputs by Meis, Pbx, and Hox (Fig. 8b). Alternatively, if the ancestral NC enhancer did not have the $\mathrm{r} 4$ activity, then gnathostome Hoxb2 and the lamprey hox 2 NC enhancers independently evolved the ability to mediate expression in $\mathrm{r} 4$.

The loss of $\mathrm{r} 4$ activity from the Hoxa2 NC enhancer may have been mitigated by the existence of a second $\mathrm{r} 4$ enhancer, located in the exon/intronic region. We have previously shown that a region containing the lamprey hox 2 exon1, intron, and exon2 was also capable of driving reporter expression in $\mathrm{r} 4$ in lamprey embryos $^{18}$. This implies that the rhombomeric activity of this genomic region is ancestral to extant vertebrates. Hence, in lamprey hox $\alpha 2$ there are two $\mathrm{r} 4$ enhancers, which may have partially redundant/shadow activities. It is notable that while both mediate expression in $\mathrm{r} 4$ in lamprey, only the upstream enhancer drives expression in NC, which indicates that there is something unique about the upstream enhancer that helps to potentiate its activity in both rhombomeres and NC.

The close proximity and partial overlap between the $\mathrm{r} 3 / \mathrm{r} 5$ and $\mathrm{r} 4 / \mathrm{NC}$ enhancers in hox 2 , in a manner analogous to its gnathostome counterparts (Fig. 1), suggests that some components of these cis-elements may be required for expression in both tissues. Our experiments with the lamprey hoxa2 enhancer showed that deletion of the Meis and Pbx-Hox sites not only led to the loss of $\mathrm{r} 4$ and $\mathrm{NC}$ expression but also to the loss of $\mathrm{r} 3 / \mathrm{r} 5$ expression (Fig. 4j). This implies that Meis and/or Pbx-Hox factors are also involved in regulating the expression of hox $\alpha 2$ in $\mathrm{r} 3 / \mathrm{r} 5$. In this regard, the mouse Hoxa2 NC enhancer partially overlaps elements required for $\mathrm{r} 3 / \mathrm{r} 5$ activity: deletion of the Meis site in NC3 $\left(\triangle N C 3 \_1\right)$ causes the loss of NC activity and also reduces $\mathrm{r} 3 / \mathrm{r} 5$ expression when tested in mouse ${ }^{10,44}$ and zebrafish (Fig. 3c; Supplementary Figure $3 \mathrm{a}-\mathrm{b})$. Thus, the Meis site contributes to both $\mathrm{r} 3 / \mathrm{r} 5$ and $\mathrm{NC}$ activities of the Hoxa 2 enhancer. Deletion of the $P b x-H o x$ site $\left(\triangle N C 3 \_2\right)$ removes NC expression but does not influence $\mathrm{r} 3 / \mathrm{r} 5$ activity in mouse ${ }^{10}$, which may reflect the control 
a Evolution of the neural crest Hox code in vertebrates

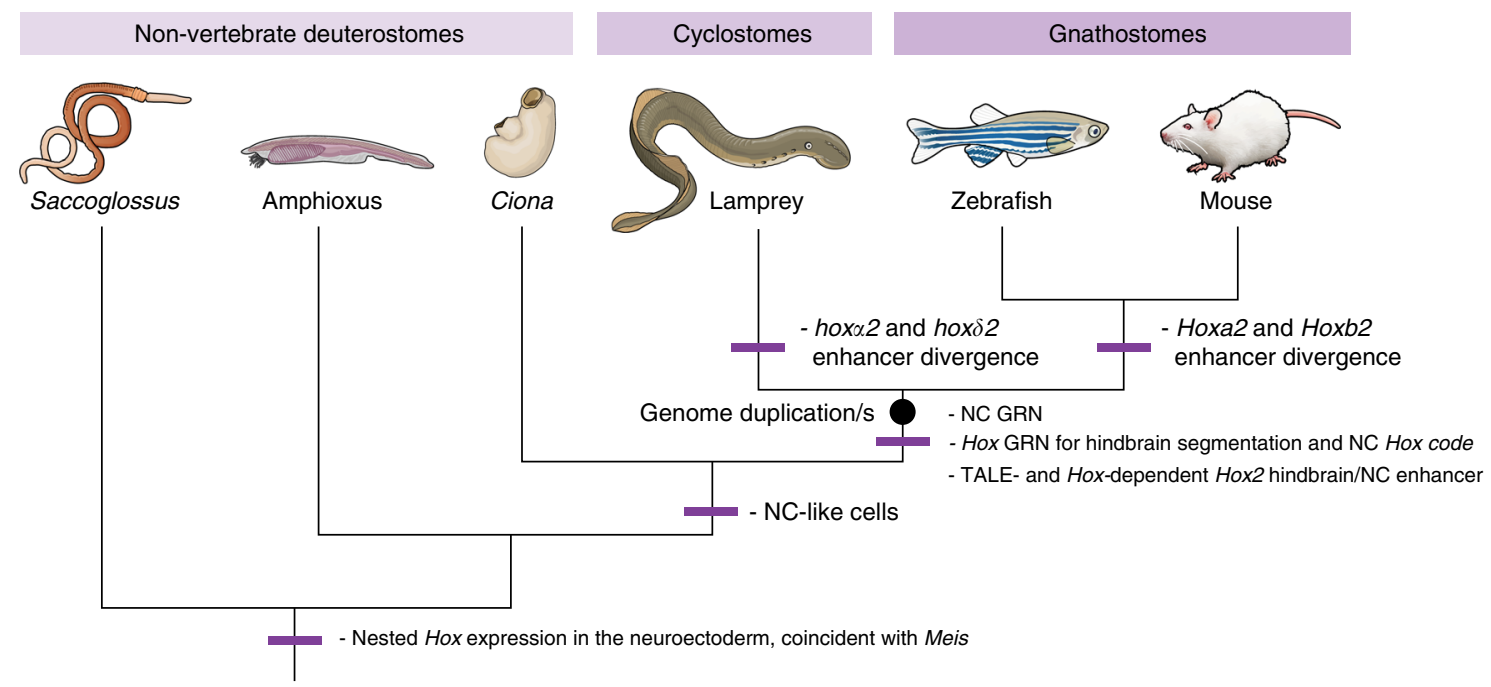

b Divergence of Hox2 hindbrain/NC enhancers

Putative ancestral Hox2 upstream enhancer activity

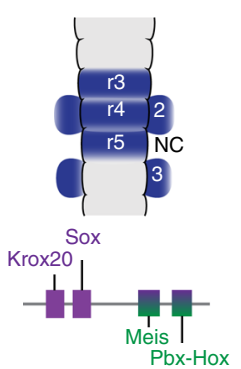

Conserved functional motifs in ancestral Hox2 upstream enhancer
Lamprey hox2 enhancers
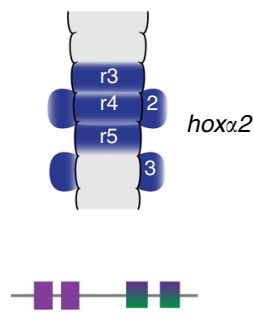

$\rightarrow+$

No anterior NC or $r 4$ activity
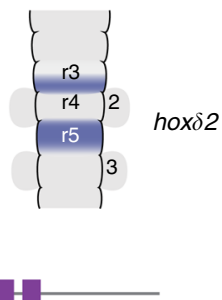

Mouse Hox2 enhancers
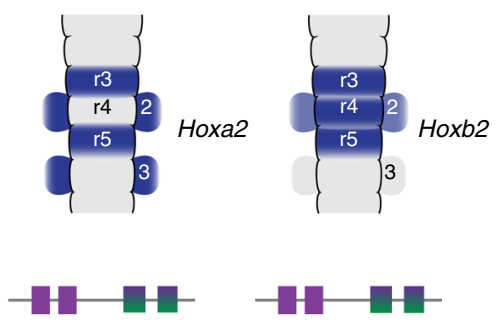

No r4 activity

Nosterior to PA2

Fig. 8 Evolutionary model for regulation of Hox2 genes in vertebrates. a A model for evolution of the neural crest (NC) Hox code, based on our data. a NC gene regulatory network (GRN) and NC Hox -code evolved in ancestral vertebrates and are conserved between cyclostomes and gnathostomes. In ancestral vertebrates, Hox2 NC expression was regulated by TALE (Three-Amino-Acid-Loop-Extension) and Hox factors, through a putative ancestral enhancer with shared NC and hindbrain activities. $\mathbf{b}$ A model for the divergence of lamprey and mouse Hox2 NC/hindbrain enhancers. Enhancer activity domains are depicted in blue in schematic dorsal views of the hindbrain and pharyngeal arches. Conserved functional motifs (Krox20, Sox, Meis, Pbx-Hox) present upstream of lamprey and mouse Hox 2 genes are shown. Lamprey and mouse enhancers show divergent activities. Comparison between expression domains and conserved motifs leads us to suggest that a putative ancestral vertebrate Hox 2 enhancer contained cis-elements for $r 3 / r 5$ expression (Krox 20 , Sox) and $\mathrm{r}$ /NC expression (Meis, Pbx-Hox). These scenarios assume that duplication events that gave rise to four Hox clusters in early vertebrates occurred prior to the cyclostome/gnathostome split, as the most parsimonious explanation 22,81 . However, it is also possible that independent genome duplication events may have occurred in cyclostome and gnathostome lineages (see Holland and Ocampo Daza ${ }^{82}$ for a recent discussion)

of r3/r5 activity being Hox-independent. For Hoxb2, r3/r5 activity appears to be independent of the Meis and $P b x-H o x$ sites ${ }^{17,45}$, suggesting that Hoxa2 and Hoxb2 enhancers have also diverged in their degree of dependence on Meis sites for $\mathrm{r} 3 / \mathrm{r} 5$ activity.

Grafting experiments in gnathostome embryos have revealed roles for both maintenance of neural tube Hox expression (pre-

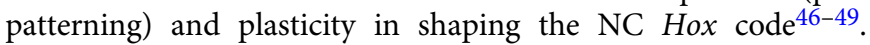
Initial AP Hox patterning in the neural tube plays an instructive role in establishing NC Hox expression, which is then modulated by permissive signals in the PA environments. Hence, the current model is that Hox expression initiated in the neural tube is not simply passively retained by migrating NC cells. The characterization of essential sites bound by Hoxa2, Meis, and $\mathrm{Pbx}$ in the mouse Hoxa2 and Hoxb2 NC enhancers suggests that Hox and TALE-dependent auto-/cross-regulation may provide a mechanism for potentiating Hox 2 expression that is set up in the pre- migratory NC. Such auto-regulation has been shown for rhombomeric expression of many Hox genes ${ }^{11,13,15}$ and may also be a general mechanism for pre-patterning the NC. Intriguingly, there appears to be context-dependent inputs that modulate the ability of Hox-response elements containing Meis and Pbx-Hox sites to potentiate activity in the hindbrain versus NC. For example, like the Hoxb2 NC/r4 enhancer, Hoxb1 has an auto-/cross-regulatory element dependent on Meis and Pbx-Hox sites, but it only mediates the expression in $\mathrm{r} 4$ and not in $\mathrm{r} 4$-derived $\mathrm{NC}^{13}$. Similarly, since Hoxa2 is expressed in $\mathrm{r} 2$ but not in $\mathrm{r} 2$-derived $\mathrm{NC}^{46}$, other regulatory mechanisms presumably prevent Hox expression in the r2-derived NC. This could include fibroblast growth factor signalling from the isthmus, which plays an important role in patterning $\mathrm{PA}^{47}$. Further regulatory analyses will be required to elucidate the generality of Hox auto-/crossregulation in NC. 
The emergence of the NC during vertebrate evolution provides a key example of how regulatory codes coevolved with novel cell types in an animal body plan. Non-vertebrate deuterostomes, like the hemichordate Saccoglossus and the cephalochordate amphioxus, lack NC but deploy nested AP domains of Hox expression to pattern their nervous system ${ }^{50-52}$. This raises the intriguing possibility that the NC Hox code in ancestral vertebrates evolved from the transfer of a deuterostome neural Hox prepattern ${ }^{14}$. Alternatively, the NC Hox code may have arisen independently, by evolution of new regulatory inputs into Hox genes. A further possibility invokes a combination of both, with shared inputs creating a Hox prepattern and independent inputs evolving to modulate this in a tissue-specific manner. Our investigation of ancestral Hox2 NC regulation in vertebrates sets the stage for examining the emergence of Hox regulation in NC during chordate evolution. This requires comparison of deuterostome development, with a focus on non-vertebrate deuterostome cell types that may be evolutionarily related to $\mathrm{NC}^{1}$.

Studies in tunicates and cephalochordates suggest that they employ similar gene regulatory programs to specify the neural plate border ${ }^{53-55}$. Recent studies in tunicates, the vertebrate sister group (Fig. 8a), have identified certain embryonic cell populations that display some characteristics of NC cells. For example, in Ecteinascidia turbinata, a colonial tunicate, the trunk lateral cells originate beside the neural tube and migrate to give rise to pigmented cell types, leading to their designation as NC-like cells ${ }^{56}$. Trunk lateral cells are also identifiable in Ciona, where they express homologues of some key genes of the vertebrate NCGRN, including Tfap $2 \alpha$ and Twist ${ }^{57}$. However, the homology of trunk lateral cells to NC has been called into question ${ }^{58}$.

Ciona Hox genes are dispersed across two chromosomes and residual spatial colinearity of expression in the nervous system has been detected for some of them ${ }^{59}$. This may be a general feature of tunicates, with similar Hox cluster disintegration seen in species from other tunicate classes ${ }^{60,61}$. Comparison with amphioxus, which has a single Hox cluster and nested colinear Hox expression along the AP axis of the neuroepithelium ${ }^{51,52,62}$, suggests that tunicates are relatively divergent in terms of their Hox genomic content and expression. Ciona intestinalis Hox 2 expression has not been detected in the developing neural tube or neural plate border, but has been described in the larval ectodermal atrial primordia ${ }^{63}$. No defects in larval morphogenesis were detected upon morpholino-mediated knockdown of Hox2 in Ciona embryos ${ }^{63}$, so the roles of Hox2 in tunicate embryonic patterning and its placement in tunicate developmental GRNs remain unclear.

In other non-vertebrate deuterostomes, the coincident expression of Hox and Meis genes in Saccoglossus ${ }^{50,64}$ and amphioxus neuroectoderm ${ }^{52,65}$ leads us to speculate that these factors may have comprised an ancestral deuterostome regulatory circuit involved in neuroectodermal patterning (Fig. 8a). Upon evolution of NC, pre-existing auto- and cross-regulatory interactions within this network may have served to maintain expression of these factors in the migrating NC. Investigation of regulatory interactions between Hox and TALE genes in invertebrate deuterostomes, combined with characterisation of the cisregulatory elements involved, could help to address whether such Hox-TALE interactions were ancestral to deuterostomes and were employed in coupling Hox genes to NC during chordate evolution. Amphioxus lacks $\mathrm{NC}^{54}$, but interspecies regulatory analysis, assaying activity of regions of the amphioxus Hox cluster in chicken and mouse embryos, revealed that some cis-elements are capable of mediating reporter expression in the hindbrain, placodes, and $\mathrm{NC}^{66}$. The activity of these elements in amphioxus is unknown but it will be important to investigate whether these represent ancestral neural elements with a capacity for mediating NC activity in vertebrates.
In summary, our finding of functionally conserved Meis and $P b x$-Hox sites in lamprey and gnathostome Hox $2 \mathrm{NC}$ enhancers focuses attention on the role of these factors in NC development. Meis and Pbx play important roles in patterning diverse tissues during development, including the hindbrain and NC, some of which may be independent of $\operatorname{Hox}^{30-33}$. For example, mouse embryos with a conditional deletion of Meis2 in NC display abnormalities in patterning the bones and connective tissue in PA1, where Hox genes are not expressed ${ }^{32}$. Hence, they could also serve as cofactors for other transcription factors ${ }^{34}$, or have independent roles in patterning NC. Therefore, while they have not been linked to the current NC-GRN, TALE factors (Pbx and Meis) may be important components in this network. If so, these transcription factors could be part of a mechanism that couples Hox genes to the NC GRN in vertebrate evolution. The conserved expression of Meis genes in NC from gnathostomes and lamprey is consistent with an ancestral role in NC and their roles and interactions in NC development require further study.

\section{Methods}

Sequence alignment. Global sequence alignment of Hox genomic loci (Fig. 3a) was performed using Multi-LAGAN ${ }^{28}$, with human as the baseline sequence and conserved sequences defined by $60 \%$ conservation over $40 \mathrm{bp}$. Sequence alignments of Hox2 enhancers (Figs. 4f, 5a, b; Supplementary Figures 3, 5, 6) were performed using AlignX in VectorNTI (Life Technologies).

Enhancer elements. Enhancer elements were selected from the published data or based on sequence conservation in cross-species alignments. The DNA for each element was amplified by PCR from genomic DNA or from pre-existing plasmids using KOD Hot Start Master Mix (Novagen). The following primers were used for amplification. The sequences in uppercase represent homology to genomic DNA and adaptor sequences for cloning are in lowercase text. References are given for primary literature in which the enhancers were identified.

crestin-1 $k b(z f)^{26}$,

F:5'-ccctcgaggtcgacGCTGAAATCTTGGGCATCTC-3';

R: $5^{\prime}$-gaggatatcgagctcGCTGGGTTACTGAGGTGAC-3';

crestin-296 bp $p^{26}$,

F: 5'-aggtaatgagggccCCGCAGATGTTCTAGTACCC-3';

R: $5^{\prime}$-gaggatatcgagctcgGGGTTAAAACAACACATTGATTAACCTGG-3';

Hoxa2(m) $\triangle N C 3-1 / \triangle N C 3-2^{10}$,

F: 5'-agggtaatgagggcccAGATCTGAATGCTGGAGC-3';

R: $5^{\prime}$-tcgccettcatagcctcgagGGTACCTTCTCTCCCTCAAAC-3';

hox $\alpha 2$ elementA,

F: 5'-agggtaatgagggccCCATCGACATGTAAACGTGGG-3';

R: $5^{\prime}$-tcctacgtcactggcGAGTAAGCGAGGTCGTGG-3'.

The following enhancer elements were cloned into the Hugo's lamprey construct (HLC) reporter vector in a previous study focusing on the hindbrain: ${ }^{18}$ hoxa2b(zf), Hoxa2a(f), Hoxa2(m), and hox $2-4 \mathrm{~kb}$.

Generation of reporter constructs. The HLC vector was created in a previous study $^{18}$. PCR-amplified enhancer elements were purified using the QIAquick PCR Purification Kit (Qiagen) and cloned into HLC by Gibson Assembly using the Gibson Assembly Master Mix (NEB). The mouse $c$-Fos promoter was cloned into the hox $\alpha 2-4 k b-H L C$ vector that had been linearized either by NcoI (for hox $\alpha 2-4$ $k b c f o s V 1$ ) or by $A s c \mathrm{I}$ and $\mathrm{NcoI}$ (for hoxa $2-4 k b$ ffosV2). The following primer pairs were used to amplify the mouse $c$-Fos promoter from a plasmid template: hox $2-4 k b$ cfos 1 ,

F: 5'-ctccgtcaaggcagcCCAGTGACGTAGGAAGTCCATC-3';

$R: 5^{\prime}$-ctcgccttgctcaccatggTGGCGACCGGTGGATCCT-3';

hox $\alpha 2-4 k b$ cfos 2 ,

F: 5'-cgcctattggctgggCCAGTGACGTAGGAAGTCCATC-3';

R: $5^{\prime}$-ctcgcccttgctcaccatggTGGCGACCGGTGGATCCT-3'.

Site-directed mutagenesis of enhancers was achieved by Gibson Assembly. For each mutation variant, two partially overlapping amplicons (left (L) and right (R)) containing the desired mutation were generated by PCR and then assembled into the linearized $H L C$ vector by 3 -fragment assembly. The following primers were used.

crestin-296 bp $\Delta$ Sox 10,

L_F: 5'-agggtaatgagggccCCGCAGATGTTCTAGTACCC-3';

L_R: $5^{\prime}$-ctagagatcgtcgcaTCTCTACGAAATTGTGCTTCTAGCAG-3';

R_F: 5'-cgtagagatgcgacGATCTCTAGAAACATTAATGCATATGAACAAAAGC-3';

$R \_R: 5^{\prime}$-gaggatatcgagctcgGGGTTAAAACAACACATTGATTAACCTGG-3'; crestin-296 bp $\Delta$ Tfap $2 \alpha$,

L_F: $5^{\prime}$-agggtaatgagggccCCGCAGATGTTCTAGTACCC-3';

L_R: 5' -ggatgtgcttaagttGAGCACATGACCAGGAGTC-3'; 
R_F:5'-atgtgctcaacttaaGCACATCCTGCTAGAAGCAC-3';

R_R: 5'-gaggatatcgagctcgGGGTTAAAACAACACATTGATTAACCTGG-3'; crestin-296 bp $\Delta M y c$,

L_F: 5'-agggtaatgagggccCCGCAGATGTTCTAGTACCC-3';

L_R: $5^{\prime}$-aaggcgagtctagaACCAGGAGTCAATTAAAAGTCTCGTG-3';

R_F:5'-ctcctggttctagaCTCGCCTTGGGCACATCC-3';

$R \_R: 5^{\prime}$-gaggatatcgagctcgGGGTTAAAACAACACATTGATTAACCTGG-3';

hox $22-4 k b \Delta k r o x 20$,

L_F: 5'-agggcccgggatcccTCGAGCCTGCAGGAAGCTTAAG-3';

L_R: 5'-cccgtaaGCGGGACTCTGTTATCTCC-3';

R_F: 5'-agtcccgcTTACCGGGATTCCGGCCAC-3';

$R \_R: 5^{\prime}$-tctacgacgacgacgacgtcgaggTCGACGCAAAGAAGCCGG-3';

hox $2-4 k b \triangle N C 3$,

L_F: 5'-agggcccgggatcccTCGAGCCTGCAGGAAGCTTAAG-3'

L_R: 5'-ccctcgctCTTGCCCTGCACAAATACTCAG-3';

R_F: 5'-agggcaagAGCGAGGGGCTCCGGAAAG-3';

$R \_R$ : 5'-tctacgacgacgacgacgtcgaggtcgaCGCAAAGAAGCCGGCCCC-3'.

Zebrafish and lamprey experiments. This study was conducted in accordance with the Guide for the Care and Use of Laboratory Animals of the National Institutes of Health and protocols were approved by the Institutional Animal Care and Use Committees of the Stowers Institute (zebrafish, RK Protocol \#2015-0149 and Protocol \#2018-0184) and California Institute of Technology (lamprey, Protocol \#1436-17)

Zebrafish reporter assay. The wild-type Slusarski $\mathrm{AB}$ zebrafish line was used for embryo micro-injection experiments using Tol2-mediated transgenesis ${ }^{67}$. A standard injection mix containing $25 \mathrm{ng} \mathrm{\mu l}^{-1}$ reporter plasmid (generated by miniprep), $35 \mathrm{ng} \mathrm{\mu l}^{-1}$ Tol2 transposase messenger RNA, and $0.05 \%$ phenol red was micro-injected into one-celled embryos at an injection volume of 3-5 nl. Embryos were screened at $24-30 \mathrm{~h}$ post fertilization for fluorescent reporter expression using a Leica M205FA microscope. In assaying reporter constructs by transient transgenesis, for each injected construct the tissue-specific GFP expression domains were noted, along with the number and proportion of screened embryos exhibiting GFP expression in each of those domains. The empty HLC reporter vector (without an enhancer) directs weak mosaic GFP expression in multiple cell types including neurons and muscle cells (Supplementary Figure 7a). The following pre-existing transgenic reporter lines were used for this study: $T g(d r . h o x a 2 b: e G F P), T g(f r$. Hoxa2a:eGFP $)^{12,18}$. The line $\operatorname{Tg}(m m$.Hoxa2b:eGFP) was generated in this study from a founder that had been micro-injected with $\operatorname{Hoxa}(m)-H L C$. Fluorescent and bright-field imaging were performed with Leica DFC360FX and DFC405C cameras and LAS AF imaging software. Images were cropped and altered for brightness and contrast using Adobe Photoshop CS5.1.

Zebrafish crestin reporter expression was analysed using $\mathrm{Tg} 2(-4.5$ crestin: EGFP) that uses the core long terminal repeat (LTR) elements of crestin located $-4.5 \mathrm{~kb}$ upstream of the putative crestin open reading frame ${ }^{26}$. Transient assays for mutated transcription factor binding sites in lamprey were performed using the minimal 296 bp crestin LTR element with the previously reported mutations as tested in zebrafish ${ }^{26}$

Lamprey reporter assay. Lamprey transient transgenesis was performed using $P$. marinus embryos at the one-cell stage and I-SceI meganuclease-mediated transgenesis ${ }^{18,68}$. Injection mixes containing $20 \mathrm{ng} \mu^{-1}$ reporter plasmid (generated by miniprep), $1 \times$ CutSmart buffer (NEB), and $0.5 \mathrm{U}_{\mu 1}^{-1} \mathrm{I}$-SceI enzyme (NEB) in water were incubated at $37^{\circ} \mathrm{C}$ for $30 \mathrm{~min}$ prior to micro-injection at a volume of $\sim 2 \mathrm{nl}$ per embryo. Embryos were screened for fluorescent reporter expression using a Zeiss SteREO Discovery V12 microscope. For each injected construct, the tissuespecific GFP expression domains were noted, along with the number and proportion of screened embryos exhibiting GFP expression in each of those domains. The empty HLC reporter vector (without an enhancer) directs GFP expression in ectoderm, yolk cells, as well as in cells dorsal to the yolk (Supplementary Figure $7 \mathrm{~b}$ ). Since transient reporter assays generate mosaic reporter expression patterns, variation in levels and domains of GFP expression are observed between embryos. For imaging we selected embryos with GFP-expressing patterns representative of the expression potential of the reporter construct, as inferred from screening more than 100 injected embryos. GFP-expressing embryos were imaged using a Zeiss SteREO Discovery V12 microscope and a Zeiss Axiocam MRm camera with AxioVision Rel 4.6 software. Images were cropped and altered for brightness using Adobe Photoshop CS5.1. Selected GFP-expressing embryos were fixed in MEMFA and dehydrated in methanol for in situ hybridisation.

Cloning lamprey in situ hybridisation probes. Probes were designed based on characterised or predicted gene sequences ${ }^{22}$, amplified from $P$. marinus genomic DNA or st18-26 embryonic complementary DNA by PCR using KOD Hot Start Master Mix (Novagen) and cloned into the $p C R 4$-TOPO vector (Life Technologies). The size of each amplified fragment is indicated (in bp). The following primers were used for PCR:

hox $\beta 1$ (674 bp, partial 3'-untranslated region),

F: $5^{\prime}$-ATGCTCCCTCAACTCCATCC-3';

R: 5'-TGACCTCTTCTCGCATGTAAGA-3'; hox $\delta 2$ (585 bp, exonic),

F: 5'-ACCTCTGCGCGACTCCTC-3';

R: 5'-CCAGACCTCCTCCTCCTCT-3';

meisC (573 bp, exonic),

F: $5^{\prime}$-CTTTGAGAAGTGCGAGCTGG-3':

R: 5'-GAAAATGCCGCGCTTCTTCT-3'.

eGFP, hox 2 , and hox 3 probe sequences were previously reported ${ }^{18}$

Lamprey in situ hybridisation. Digoxygenin-labelled probes were generated by standard methods and purified using the MEGAclear Transcription Clean-Up Kit (Ambion). Lamprey embryos were staged according to Tahara et al. ${ }^{69}$. Lamprey whole-mount in situ hybridisation was performed on MEMFA-fixed embryos following established protocols ${ }^{70}$, with the following additions to the protocol: ${ }^{71}$ methanol-stored embryos were transferred into ethanol and left overnight prior to rehydration; embryos were treated with $0.5 \%$ acetic anhydride in $0.1 \mathrm{M}$ triethanolamine after proteinase $\mathrm{K}$ treatment. For imaging, embryos were cleared in benzyl alcohol:benzyl benzoate and mounted in Permount (Fisher Scientific).

For sectioning after in situ hybridisation, embryos were transferred into $30 \%$ sucrose in phosphate-buffered saline, embedded in O.C.T. Compound and sectioned to $10-\mu \mathrm{m}$-thick cryosections. Images were taken using a Zeiss Axiovert 200 microscope with AxioCam HRc camera and AxioVision Rel 4.8.2 software.

ES cell culture. ES cells were cultured in feeder-free conditions using N2B27 +2 media supplemented with $2000 \mathrm{U} \mathrm{mL}^{-1}$ of ESGRO (Millipore) on a gelatinized plate. KH2 ES cells ${ }^{72}$ with epitope-tagged Hoxb1 (3XFLAG-MYC) were used for Hoxb1 ChIP using anti-flag antibody (F1804-Sigma). Unmodified KH2 cell lines were used for ChIP experiments for Pbx (SC-888; Santa Cruz), Meis (SC-25412; Santa Cruz), Prep1 (ab55603; Abcam), Prep2 (sc-292315X; Santa Cruz) and EP300 (Sc-585X; Santa Cruz). Cells were differentiated to neuroectoderm in differentiation media containing DMEM $+10 \%$ (vol/vol) Serum + NEAA $+3 \mu$ M RA for a requisite length of time. Cells were harvested at $80-90 \%$ confluency.

Chromatin immunoprecipitation-sequencing. ChIP-seq was performed according to the Upstate protocol as described ${ }^{73}$ with modifications. Cells were fixed with $1 \%$ formaldehyde by incubating at $37^{\circ} \mathrm{C}$ for $11 \mathrm{~min}$. The reaction was quenched for $5 \mathrm{~min}$ by the addition of $1 / 10$ th volume of $1.25 \mathrm{M}$ glycine. Cells were sonicated for $25 \mathrm{~min}$ in a Bioruptor at high setting and $30 \mathrm{~s}$ on-off cycle. Respective antibodies attached to sepharose-A beads were used for immunoprecipitation. Sequencing of ChIP-seq libraries was performed on the Illumina HiSeq 2500, 51 bp single end. Raw reads were aligned to the UCSC mm10 mouse genome with bowtie2 $2.2 .0^{74}$ Primary reads from each bam were normalized to reads per million and bigWig tracks visualized at the UCSC genome browser (https://genome.ucsc.edu/).

Assay for transposase-accessible chromatin-sequencing. Assay for transposase-accessible chromatin-sequencing was performed as described previously ${ }^{75}$. Fifty thousand cells were counted using the sceptre 2.0 cell counter (EMD Millipore). The tagmentation reaction was performed using the Nextera DNA Library Prep Kit (Illumina, FC-121-1030) and libraries indexed using the Nextera Index Kit (Illumina, FC-121-1011). Libraries were size selected by Bluepippin (Sage Science) and sequenced on Illumina HiSEq. 2500 instrument. Following sequencing, Illumina Real Time Analysis v1.18.64 and CASAVA v1.8.2 were run to demultiplex reads and generate FASTQ files.

Reporting summary. Further information on experimental design is available in the Nature Research Reporting Summary linked to this article.

\section{Data availability}

The authors declare that all data supporting the findings of this study are available within the article and its supplementary information files or from the corresponding author upon reasonable request. All raw sequencing data from this study underlying Fig. 7a have been deposited in the NCBI BioProject database [https://www.ncbi.nlm.nih.gov/ bioproject] under accession code PRJNA341679 and Sequence Read Archive under accession code SRP079975 and PRJNA503882. Original data underlying this manuscript can be accessed from the Stowers Original Data Repository at [http://odr.stowers.org/ websimr/]. A reporting summary for this Article is available as a Supplementary Information file.

Received: 5 December 2018 Accepted: 26 February 2019 Published online: 13 March 2019

\section{References}

1. Green, S. A., Simoes-Costa, M. \& Bronner, M. E. Evolution of vertebrates as viewed from the crest. Nature 520, 474-482 (2015).

2. Le Douarin, N. \& Kalcheim, C. The Neural Crest 2nd edn (Cambridge University Press, Cambridge, 1999). 
3. Northcutt, R. The new head hypothesis revisited. J. Exp. Zool. B 304, 274-297 (2005).

4. Parker, H. J., Pushel, I. \& Krumlauf, R. Coupling the roles of Hox genes to regulatory networks patterning cranial neural crest. Dev. Biol. https://doi.org/ 10.1016/j.ydbio.2018.1003.1016 (2018).

5. Gavalas, A., Trainor, P., Ariza-McNaughton, L. \& Krumlauf, R. Synergy between Hoxal and Hoxb1: the relationship between arch patterning and the generation of cranial neural crest. Development 128, 3017-3027 (2001).

6. Martik, M. L. \& Bronner, M. E. Regulatory logic underlying diversification of the neural crest. Trends Genet 33, 715-727 (2017).

7. Sauka-Spengler, T., Meulemans, D., Jones, M. \& Bronner-Fraser, M. Ancient evolutionary origin of the neural crest gene regulatory network. Dev. Cell 13, 405-420 (2007).

8. Minoux, M. \& Rijli, F. M. Molecular mechanisms of cranial neural crest cell migration and patterning in craniofacial development. Development 137, 2605-2621 (2010).

9. Kitazawa, T. et al. Distinct effects of Hoxa2 overexpression in cranial neural crest populations reveal that the mammalian hyomandibular-ceratohyal boundary maps within the styloid process. Dev. Biol. 402, 162-174 (2015).

10. Maconochie, M. et al. Regulation of Hoxa2 in cranial neural crest cells involves members of the AP-2 family. Development 126, 1483-1494 (1999).

11. Maconochie, M. K. et al. Cross-regulation in the mouse HoxB complex: the expression of Hoxb2 in rhombomere 4 is regulated by Hoxb1. Genes Dev. 11, 1885-1896 (1997).

12. McEllin, J. A., Alexander, T. B., Tumpel, S., Wiedemann, L. M. \& Krumlauf, R. Analyses of fugu hoxa2 genes provide evidence for subfunctionalization of neural crest cell and rhombomere cis-regulatory modules during vertebrate evolution. Dev. Biol. 409, 530-542 (2016).

13. Parker, H. J. \& Krumlauf, R. Segmental arithmetic: summing up the Hox gene regulatory network for hindbrain development in chordates. Wiley Interdiscip. Rev. Dev. Biol. 6., https://doi.org/10.1002/wdev.286(2017).

14. Wada, H., Escriva, H., Zhang, S. \& Laudet, V. Conserved RARE localization in amphioxus Hox clusters and implications for Hox code evolution in the vertebrate neural crest. Dev. Dyn. 235, 1522-1531 (2006).

15. Tümpel, S. et al. Expression of Hoxa2 in rhombomere 4 is regulated by a conserved cross-regulatory mechanism dependent upon Hoxb1. Dev. Biol. 302, 646-660 (2007).

16. Lampe, $\mathrm{X}$. et al. An ultraconserved Hox-Pbx responsive element resides in the coding sequence of Hoxa2 and is active in rhombomere 4. Nucleic Acids Res. 36, 3214-3225 (2008).

17. Ferretti, E. et al. Segmental expression of Hoxb2 in $\mathrm{r} 4$ requires two separate sites that integrate cooperative interactions between Prep1, Pbx and Hox proteins. Development 127, 155-166 (2000).

18. Parker, H. J., Bronner, M. E. \& Krumlauf, R. A Hox regulatory network of hindbrain segmentation is conserved to the base of vertebrates. Nature $\mathbf{5 1 4}$ 490-493 (2014).

19. Green, S. A., Uy, B. R. \& Bronner, M. E. Ancient evolutionary origin of vertebrate enteric neurons from trunk-derived neural crest. Nature 544, 88-91 (2017).

20. Shimeld, S. M. \& Donoghue, P. C. Evolutionary crossroads in developmental biology: cyclostomes (lamprey and hagfish). Development 139, 2091-2099 (2012).

21. Mehta, T. K. et al. Evidence for at least six Hox clusters in the Japanese lamprey (Lethenteron japonicum). Proc. Natl Acad. Sci. USA 110, 16044-16049 (2013).

22. Smith, J. J. et al. The sea lamprey germline genome provides insights into programmed genome rearrangement and vertebrate evolution. Nat. Genet. 50, 270-277 (2018).

23. Pascual-Anaya, J. et al. Hagfish and lamprey Hox genes reveal conservation of temporal colinearity in vertebrates. Nat. Ecol. Evol. 2, 859-866 (2018).

24. Takio, Y. et al. Evolutionary biology: lamprey Hox genes and the evolution of jaws. Nature 429, 1-2 (2004).

25. Takio, Y. et al. Hox gene expression patterns in Lethenteron japonicum embryos-insights into the evolution of the vertebrate Hox code. Dev. Biol. 308, 606-620 (2007).

26. Kaufman, C. K. et al. A zebrafish melanoma model reveals emergence of neural crest identity during melanoma initiation. Science 351, aad2197 (2016).

27. Nonchev, S. et al. Segmental expression of Hoxa-2 in the hindbrain is directly regulated by Krox-20. Development 122, 543-554 (1996).

28. Brudno, M. et al. LAGAN and Multi-LAGAN: efficient tools for large-scale multiple alignment of genomic DNA. Genome Res. 13, 721-731 (2003).

29. Burglin, T. R. Analysis of TALE superclass homeobox genes (MEIS, PBC KNOX, Iroquois, TGIF) reveals a novel domain conserved between plants and animals. Nucleic Acids Res. 25, 4173-4180 (1997).

30. Moens, C. B. \& Selleri, L. Hox cofactors in vertebrate development. Dev. Biol. 291, 193-206 (2006).

31. Choe, S. K., Vlachakis, N. \& Sagerstrom, C. G. Meis family proteins are required for hindbrain development in the zebrafish. Development 129, 585-595 (2002).
32. Machon, O., Masek, J., Machonova, O., Krauss, S. \& Kozmik, Z. Meis2 is essential for cranial and cardiac neural crest development. BMC Dev. Biol. 15 40 (2015).

33. Deflorian, G. et al. Prep1.1 has essential genetic functions in hindbrain development and cranial neural crest cell differentiation. Development 131, 613-627 (2004)

34. Laurent, A., Bihan, R., Omilli, F., Deschamps, S. \& Pellerin, I. PBX proteins: much more than Hox cofactors. Int. J. Dev. Biol. 52, 9-20 (2008).

35. Tümpel, S., Maconochie, M., Wiedemann, L. M. \& Krumlauf, R. Conservation and diversity in the cis-regulatory networks that integrate information controlling expression of Hoxa2 in hindbrain and cranial neural crest cells in vertebrates. Dev. Biol. 246, 45-56 (2002).

36. Amin, S. et al. Hoxa2 selectively enhances Meis binding to change a branchial arch ground state. Dev. Cell 32, 265-277 (2015)

37. Donaldson, I. J. et al. Genome-wide occupancy links Hoxa2 to Wnt- $\beta$-catenin signaling in mouse embryonic development. Nucleic Acids Res 40, 3990-4001 (2012).

38. Merabet, S. \& Mann, R. S. To be specific or not: the critical relationship between Hox and TALE proteins. Trends Genet. 32, 334-347 (2016).

39. De Kumar, B. et al. Analysis of dynamic changes in retinoid-induced transcription and epigenetic profiles of murine Hox clusters in ES cells. Genome Res. 25, 1229-1243 (2015).

40. De Kumar, B. et al. HOXA1 and TALE proteins display cross-regulatory interactions and form a combinatorial binding code on HOXA1 targets. Genome Res. 27, 1501-1512 (2017).

41. De Kumar, B. et al. Dynamic regulation of Nanog and stem cell-signaling pathways by Hoxa1 during early neuro-ectodermal differentiation of ES cells. Proc. Natl Acad. Sci. USA 114, 5838-5845 (2017).

42. De Kumar, B. et al. Hoxal targets signaling pathways during neural differentiation of ES cells and mouse embryogenesis. Dev. Biol. 432, 151-164 (2017).

43. Hudry, B. et al. Molecular insights into the origin of the Hox-TALE patterning system. Elife 3, e01939 (2014).

44. Maconochie, M. K., Nonchev, S., Manzanares, M., Marshall, H. \& Krumlauf, R. Differences in Krox20-dependent regulation of Hoxa2 and Hoxb2 during hindbrain development. Dev. Biol. 233, 468-481 (2001).

45. Sham, M. H. et al. The zinc finger gene Krox-20 regulates Hoxb-2 (Hox2.8) during hindbrain segmentation. Cell 72, 183-196 (1993)

46. Prince, V. \& Lumsden, A. Hox-a2 expression in normal and transposed rhombomeres: independent regulation in the neural tube and neural crest. Development 120, 911-923 (1994).

47. Trainor, P. A., Ariza-McNaughton, L. \& Krumlauf, R. Role of the isthmus and FGFs in resolving the paradox of neural crest plasticity and prepatterning. Science 295, 1288-1291 (2002).

48. Trainor, P. \& Krumlauf, R. Plasticity in mouse neural crest cells reveals a new patterning role for cranial mesoderm. Nat. Cell Biol. 2, 96-102 (2000).

49. Schilling, T. F., Prince, V. \& Ingham, P. W. Plasticity in zebrafish hox expression in the hindbrain and cranial neural crest. Dev. Biol. 231, 201-216 (2001).

50. Aronowicz, J. \& Lowe, C. J. Hox gene expression in the hemichordate Saccoglossus kowalevskii and the evolution of deuterostome nervous systems. Integr. Comp. Biol. 46, 890-901 (2006).

51. Wada, H., Garcia-Fernandez, J. \& Holland, P. W. Colinear and segmental expression of amphioxus Hox genes. Dev. Biol. 213, 131-141 (1999).

52. Schubert, M., Holland, N. D., Laudet, V. \& Holland, L. Z. A retinoic acid-Hox hierarchy controls both anterior/posterior patterning and neuronal specification in the developing central nervous system of the cephalochordate amphioxus. Dev. Biol. 296, 190-202 (2006).

53. Medeiros, D. M. The evolution of the neural crest: new perspectives from lamprey and invertebrate neural crest-like cells. Wiley Interdiscip. Rev. Dev. Biol. 2, 1-15 (2013).

54. Yu, J. K., Meulemans, D., McKeown, S. J. \& Bronner-Fraser, M. Insights from the amphioxus genome on the origin of vertebrate neural crest. Genome Res. 18, 1127-1132 (2008).

55. Denes, A. S. et al. Molecular architecture of annelid nerve cord supports common origin of nervous system centralization in bilateria. Cell 129, 277-288 (2007).

56. Jeffery, W. R., Strickler, A. G. \& Yamamoto, Y. Migratory neural crest-like cells form body pigmentation in a urochordate embryo. Nature 431, 696-699 (2004)

57. Jeffery, W. R. et al. Trunk lateral cells are neural crest-like cells in the ascidian Ciona intestinalis: insights into the ancestry and evolution of the neural crest. Dev. Biol. 324, 152-160 (2008).

58. Hall, B. K. \& Gillis, J. A. Incremental evolution of the neural crest, neural crest cells and neural crest-derived skeletal tissues. J. Anat. 222, 19-31 (2013).

59. Ikuta, T., Yoshida, N., Satoh, N. \& Saiga, H. Ciona intestinalis Hox gene cluster: Its dispersed structure and residual colinear expression in development. Proc. Natl Acad. Sci. USA 101, 15118-15123 (2004) 
60. Seo, H. C. et al. Hox cluster disintegration with persistent anteroposterior order of expression in Oikopleura dioica. Nature 431, 67-71 (2004).

61. Sekigami, Y. et al. Hox gene cluster of the ascidian, Halocynthia roretzi, reveals multiple ancient steps of cluster disintegration during ascidian evolution. Zool. Lett. 3, 17 (2017)

62. Garcia-Fernandez, J. \& Holland, P. W. H. Archetypal organisation of the amphioxus Hox gene cluster. Nature 370, 563-566 (1994).

63. Ikuta, T., Satoh, N. \& Saiga, H. Limited functions of Hox genes in the larval development of the ascidian Ciona intestinalis. Development 137, 1505-1513 (2010).

64. Lemons, D., Fritzenwanker, J. H., Gerhart, J., Lowe, C. J. \& McGinnis, W. Cooption of an anteroposterior head axis patterning system for proximodistal patterning of appendages in early bilaterian evolution. Dev. Biol. 344, 358-362 (2010).

65. Albuixech-Crespo, B. et al. Molecular regionalization of the developing amphioxus neural tube challenges major partitions of the vertebrate brain. PLoS Biol. 15, e2001573 (2017).

66. Manzanares, M. et al. Conservation and elaboration of Hox gene regulation during evolution of the vertebrate head. Nature 408, 854-857 (2000).

67. Fisher, S. et al. Evaluating the biological relevance of putative enhancers using Tol2 transposon-mediated transgenesis in zebrafish. Nat. Protoc. 1, 1297-1305 (2006).

68. Parker, H. J., Sauka-Spengler, T., Bronner, M. \& Elgar, G. A reporter assay in lamprey embryos reveals both functional conservation and elaboration of vertebrate enhancers. PLoS ONE 9, e85492 (2014).

69. Tahara, Y. Normal stages of development in the lamprey, Lampetra reissneri (Dybowski). Zool. Sci. 5, 109-118 (1988).

70. Nikitina, N., Bronner-Fraser, M. \& Sauka-Spengler, T. The sea lamprey Petromyzon marinus: a model for evolutionary and developmental biology. Cold Spring Harb. Protoc. 2009, pdb emol13 (2009).

71. Lowe, C. J., Tagawa, K., Humphreys, T., Kirschner, M. \& Gerhart, J. Hemichordate embryos: procurement, culture, and basic methods. Methods Cell Biol. 74, 171-194 (2004).

72. Beard, C., Hochedlinger, K., Plath, K., Wutz, A. \& Jaenisch, R. Efficient method to generate single-copy transgenic mice by site-specific integration in embryonic stem cells. Genesis 44, 23-28 (2006).

73. Smith, K. T., Martin-Brown, S. A., Florens, L., Washburn, M. P. \& Workman, J. L. Deacetylase inhibitors dissociate the histone-targeting ING2 subunit from the Sin3 complex. Chem. Biol. 17, 65-74 (2010).

74. Langmead, B. \& Salzberg, S. L. Fast gapped-read alignment with Bowtie 2. Nat. Methods 9, 357-359 (2012).

75. Buenrostro, J. D., Wu, B., Chang, H. Y. \& Greenleaf, W. J. ATAC-seq: a method for assaying chromatin accessibility genome-wide. Curr. Protoc. Mol. Biol. 109, 2129 21-21 2929 (2015).

76. Khan, A. et al. JASPAR 2018: update of the open-access database of transcription factor binding profiles and its web framework. Nucleic Acids Res. 46, D1284 (2018).

77. Meng, X., Brodsky, M. H. \& Wolfe, S. A. A bacterial one-hybrid system for determining the DNA-binding specificity of transcription factors. Nat. Biotechnol. 23, 988-994 (2005).

78. Badis, G. et al. Diversity and complexity in DNA recognition by transcription factors. Science 324, 1720-1723 (2009).

79. Chang, C. P. et al. Meis proteins are major in vivo DNA binding partners for wild-type but not chimeric Pbx proteins. Mol. Cell. Biol. 17, 5679-5687 (1997).

80. Lu, Q., Wright, D. D. \& Kamps, M. P. Fusion with E2A converts the Pbx1 homeodomain protein into a constitutive transcriptional activator in human leukemias carrying the $\mathrm{t}(1 ; 19)$ translocation. Mol. Cell. Biol. 14, 3938-3948 (1994).

81. Sacerdot, C., Louis, A., Bon, C., Berthelot, C. \& Roest Crollius, H. Chromosome evolution at the origin of the ancestral vertebrate genome. Genome Biol. 19, 166 (2018).
82. Holland, L. Z. \& Ocampo Daza, D. A new look at an old question: when did the second whole genome duplication occur in vertebrate evolution? Genome Biol. 19, 209 (2018).

\section{Acknowledgements}

We thank Dorit Hockman, Tetsuto Miyashita, and Megan Martik for lamprey husbandry assistance, the Stowers Institute aquatics facility for zebrafish care, and Histology facility for sectioning assistance. This study was conducted in accordance with the recommendations in the Guide for the Care and Use of Laboratory Animals of the NIH and protocols approved by the Institutional Animal Care and Use Committees of the Stowers Institute (Zebrafish, RK Protocol: \#2015-0149), California Institute of Technology (lamprey, MEB Protocol: \#1436-11), and the veterinary office of UZH and the Canton of Zürich. K.D.P., C.H., and C.M. were supported by Science Foundation (SNSF) professorship (C.M. grant 170623), a Marie Curie Career Integration Grant from the European Commission (C.M. grant PCIG14-GA-2013-631984), the Swiss Cancer League, and the Canton of Zürich. H.J.P., B.D.K., L.M.W., and R.K. were supported by the Stowers Institute (R.K. grant \#2013-1001). S.A.G. and M.E.B. were supported by grants R01NS086907 and R01DE017911.

\section{Author contributions}

H.J.P., M.E.B. and R.K. conceived this research programme. H.J.P., B.D.K., K.D.P. and C. H. conducted the experiments. S.A.G. performed lamprey husbandry. C.K.K. and C.M. developed the crestin transgenic zebrafish line and associated constructs. H.J.P., B.D.K., C.M., L.M.W., M.E.B. and R.K. analysed the data, discussed the ideas and interpretations, and wrote the manuscript.

\section{Additional information}

Supplementary Information accompanies this paper at https://doi.org/10.1038/s41467019-09197-8.

\section{Competing interests: The authors declare no competing interests.}

Reprints and permission information is available online at http://npg.nature.com/ reprintsandpermissions/

Journal peer review information: Nature Communications thanks David McCauley, Elena Silva and the other anonymous reviewer(s) for their contribution to the peer review of this work. Peer reviewer reports are available.

Publisher's note: Springer Nature remains neutral with regard to jurisdictional claims in published maps and institutional affiliations.

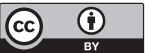

Open Access This article is licensed under a Creative Commons Attribution 4.0 International License, which permits use, sharing, adaptation, distribution and reproduction in any medium or format, as long as you give appropriate credit to the original author(s) and the source, provide a link to the Creative Commons license, and indicate if changes were made. The images or other third party material in this article are included in the article's Creative Commons license, unless indicated otherwise in a credit line to the material. If material is not included in the article's Creative Commons license and your intended use is not permitted by statutory regulation or exceeds the permitted use, you will need to obtain permission directly from the copyright holder. To view a copy of this license, visit http://creativecommons.org/ licenses/by/4.0\%

(C) The Author(s) 2019 